TRANSACTIONS OF THE

AMERICAN MATHEMATICAL SOCIETY

Volume 365, Number 3, March 2013, Pages 1441-1468

S 0002-9947(2012)05659-5

Article electronically published on September 12, 2012

\title{
THE SHORT TORIC POLYNOMIAL
}

\author{
GÁBOR HETYEI
}

\begin{abstract}
We introduce the short toric polynomial associated to a graded Eulerian poset. This polynomial contains the same information as the two toric polynomials introduced by Stanley, but allows different algebraic manipulations. The intertwined recurrence defining Stanley's toric polynomials may be replaced by a single recurrence, in which the degree of the discarded terms is independent of the rank. A short toric variant of the formula by Bayer and Ehrenborg, expressing the toric $h$-vector in terms of the $c d$-index, may be stated in a rank-independent form, and it may be shown using weighted lattice path enumeration and the reflection principle. We use our techniques to derive a formula expressing the toric $h$-vector of a dual simplicial Eulerian poset in terms of its $f$-vector. This formula implies Gessel's formula for the toric $h$-vector of a cube, and may be used to prove that the nonnegativity of the toric $h$-vector of a simple polytope is a consequence of the Generalized Lower Bound Theorem holding for simplicial polytopes.
\end{abstract}

\section{INTRODUCTION}

As mathematicians, we often look for a "magic" simplification that makes known results easier to state, and helps us find new results which were cumbersome to even talk about using the old terminology. In the study of Eulerian partially ordered sets such a wonderful simplification was the introduction of the $c d$-index by Fine (see [6]) allowing us to restate the already known Bayer-Billera formulas 2 in a simpler form and to formulate Stanley's famous nonnegativity conjecture [19] regarding the $c d$-coefficients of Gorenstein* posets, shown many years later by Karu $[13$.

The present author believes that a similar "magic" moment has yet to arrive in the study of the toric polynomials $f(P, x)$ and $g(P, x)$ associated to an Eulerian poset $\widehat{P}=P \uplus\{\widehat{1}\}$ by Stanley [20]. Without doubt, these invariants are very important, linked to deep results in algebraic topology, and yielding highly nontrivial combinatorial interpretations, whenever such interpretations were found. However, the defining intertwined recurrence is difficult to use directly, not only because two sequences of polynomials need to be defined simultaneously, but also because the degree of the terms to be discarded in the process changes all the time as the rank of the intervals considered changes.

The introduction of the new invariant proposed in this paper is probably not the desired "magic simplification" yet, but it represents a modest improvement in some cases. The idea on which it is based is very simple and could also be used beyond the confines of our current area. As explained in Section 2, there is a bijective way to associate each multiplicatively symmetric polynomial $p(x)$ (having a symmetric

Received by the editors November 16, 2010 and, in revised form, June 22, 2011.

2010 Mathematics Subject Classification. Primary 06A07; Secondary 05A15, 06A11, 52B05.

Key words and phrases. Eulerian poset, toric $h$-vector, Narayana numbers, reflection principle, Morgan-Voyce polynomial. 
array of coefficients) to an additively symmetric polynomial $q(x)$ (whose multiset of zeros is symmetric to the origin) of the same degree, having the same set of coefficients. For example, the additively symmetric variant of $1-2 x+7 x^{3}-2 x^{5}+x^{6}$ is $x^{6}-2 x^{4}+7$. There is no change when we want to extract the coefficients of the individual polynomials only, but when we consider a sequence $\left\{p_{n}(x)\right\}_{n \geq 0}$ of multiplicatively symmetric polynomials, given by some rule, switching to the additively symmetric variant $\left\{q_{n}(x)\right\}_{n>0}$ greatly changes the appearance of the rules, making them sometimes easier to manipulate. Since multiplicatively symmetric polynomials abound in combinatorics, the basic idea presented in Section 2 is worth trying in many situations, unrelated to our current subject.

The short toric polynomial $\mathrm{t}(P, x)$, associated to a graded Eulerian poset $\widehat{P}$ is defined in Section 3 as the additively symmetric variant of Stanley's toric polynomial $f(P, x)$. The intertwined recurrence defining $f(P, x)$ and $g(P, x)$ is equivalent to a single recurrence for $\mathrm{t}(P, x)$. In this recurrence, multiplication by negative powers of $x$ occurs and we obtain a polynomial by discarding all terms of negative degree and also certain constant terms. It is a tempting thought to use this recurrence to generalize the short toric polynomial to all ranked posets having a unique minimum element, even if in the cases of lower Eulerian posets, "severe loss of information" may occur, compared to Stanley's generalization of $f(P, x)$ to such posets. We state and outline the proof of the short toric variant of Fine's formula (see [1] and 3. Theorem 7.14]) expressing the toric $h$-vector in terms of the flag $f$-vector. Using this formula, it is easy to observe that the generalization of $\mathrm{t}(P, x)$ makes the most sense for ranked posets with unique minimum element $\widehat{0}$ such that the reduced Euler characteristic of the order complex of $P \backslash\{\widehat{0}\}$ is not zero.

Arguably the nicest result in this paper is Theorem 4.7 in Section 4, expressing the short toric polynomial associated to a graded Eulerian poset by defining two linear operators on the vector space of polynomials that need to be substituted into the reverse of the $c d$-index and applied to the constant polynomial 1 . The fact that the toric $h$-vector may be computed by replacing the letters $c$ and $d$ in the reverse of the $c d$-index by some linear operators and applying the resulting linear operator to a specific vector is a direct consequence of the famous result by Bayer and Ehrenborg [3, Theorem 4.2], expressing the toric $h$-vector in terms of the $c d$ index. In applications, the use of the Bayer-Ehrenborg result may be facilitated by finding a linearly equivalent presentation that is easier to manipulate. In this sense our Theorem 4.7 is analogous to Lee's result [16. Theorem 5], presenting another easily memorizable reformulation of [3, Theorem 4.2]. Our Theorem 4.7 offers the first rank-independent substitution rule, making it more useful in proofs involving induction on rank. Theorem 4.3, which is the reason behind Theorem 4.7, also implies the short toric variant of the Bayer-Ehrenborg result [3, Theorem 4.2], and has a proof using weighted lattice path enumeration and the reflection principle. The idea of using a weighted lattice path model to interpret Fine's formula is already present in the work of Bayer and Ehrenborg [3, Section 7.4] where it is used to provide an alternative proof of their formula [3, Theorem 3.1] expressing the toric $h$-vector in terms of the $a b$-index. By finding the $c d$-index via calculating the $c e$-index first (instead of the $a b$-index), and by using the short toric form, the applicability of the reflection principle becomes apparent.

The short toric variant of the above cited Bayer-Ehrenborg result highlights the importance of a sequence of polynomials $\left\{\widetilde{Q}_{n}(x)\right\}_{n \geq 0}$, a variant of the polynomials 
$\left\{Q_{n}(x)\right\}_{n \geq 0}$ already used by Bayer and Ehrenborg [3. In Section 5] we take a closer look at this basis of the vector space of polynomials, alongside the basis formed by the short toric polynomials $\left\{t_{n}(x)\right\}_{n \geq 0}$ associated to Boolean algebras. The polynomials $\left\{\widetilde{Q}_{n}(x)\right\}_{n \geq 0}$ turn out to be the dual basis to the Morgan-Voyce polynomials; whereas the polynomials $\left\{t_{n}(x)\right\}_{n \geq 0}$ may be used to provide a simple formula connecting the short toric polynomial to Stanley's toric polynomial $g(P, x)$.

The proof is always in the pudding; the usefulness of a technique is much more apparent if it is used to answer a question that was open before. Such an application may be found in Section [6] where we express the toric $h$-vector of an Eulerian dual simplicial poset in terms of its $f$-vector. This question was raised by Kalai; see [20]. Besides using Theorem 4.7, the proof of the formula depends on a formula conjectured by Stanley [19, Conjecture 3.1] and shown by the present author [10, Theorem 2 ], expressing the contribution of the $h$-vector entries of an Eulerian simplicial poset to its $c d$-index as weights of certain André permutations. The result was not found easily: it was conjectured after using Maple to compute many examples, and then shown by induction. Finding a "more combinatorial" explanation in the future is desirable; the numbers indicate that the models involving Narayana numbers are good candidates for generalization. Finally, an equivalent form of our formula shows the following, perhaps surprising result: the nonnegativity of the toric $h$-vector of a simple polytope is a direct consequence of the Generalized Lower Bound Theorem (GLBT) holding for simplicial polytopes. Thanks to Karu [12, we know that the GLBT holds for all polytopes, which yields a much stronger statement on the $h$-vector of an arbitrary simple polytope. However, the validity of the GLBT for simplicial polytopes was shown much earlier by Stanley [21]; and the nonnegativity of the toric $h$-entries of a simple polytope is derived from this earlier result using a short and elementary reasoning.

The paper re-emphasizes the close relation between the study of the toric polynomials and "Catalan combinatorics". This connection is already present in the formulas of Bayer and Ehrenborg [3. Theorems 4.1 and 4.2] expressing the toric $h$-polynomial of an Eulerian poset in terms of its flag $h$-vector and $c d$-index; the same holds for the formula of Billera and Brenti [7, Theorem 3.3] expressing the Kazhdan-Lusztig polynomial of any Bruhat interval in any Coxeter group in terms of the complete $c d$-index as well as for the formulas of the present author [11, expressing the toric $h$-contributions of cubical shelling components. The present work adds explicit relations to weighted lattice path enumeration, Morgan-Voyce polynomials and the Narayana numbers. Besides exploring these connections further, the generalization of the definition of the short toric polynomials to non-Eulerian posets is worth further investigation. A good starting point could be computing the short toric polynomial associated to the face lattice of a finite dimensional vector space of a finite field; if the outcome is unsatisfying, the example is probably a good source of inspiration to define a $q$-analogue.

\section{Preliminaries}

A partially ordered set $P$ is graded if it has a unique minimum element $\widehat{0}$, a unique maximum element $\widehat{1}$ and a rank function $\rho: P \rightarrow \mathbb{N}$ satisfying $\rho(\widehat{0})=0$ and $\rho(y)=\rho(x)+1$ whenever $y$ covers $x$. The rank of $P$ is $\rho(\widehat{1})$. An often studied invariant of a graded poset of rank $n+1$ is its flag $f$-vector $\left(f_{S}: S \subseteq[1, n]\right)$, where $f_{S}=f_{S}(P)$ is the number of maximal chains in the $S$ rank-selected subposet 
$P_{S}=\{u \in P: \rho(u) \in S\}$. Here and throughout this paper we use the interval notation $[i, j]$ to denote the set of integers $\{i, i+1, \ldots, j\}$. A graded poset is Eulerian if every interval $[u, v] \subseteq P$ with $u<v$ satisfies $\sum_{z \in[u, v]}(-1)^{\rho(z)}=0$. All linear relations satisfied by the flag $f$-vector of an Eulerian poset were given by Bayer and Billera [2. It was observed by Fine and proven by Bayer and Klapper [6] that the Bayer-Billera relations may be restated as the existence of the $c d$-index, as follows. Introducing the flag h-vector $\left(h_{S}: S \subseteq[1, n]\right)$ of a graded poset of rank $n+1$ by setting

$$
h_{S}:=\sum_{T \subseteq S}(-1)^{|S|-|T|} f_{T},
$$

we define the $a b$-index as the polynomial

$$
\Psi_{P}(a, b)=\sum_{S \subseteq[1, n]} h_{S} u_{S}
$$

in noncommuting variables $a$ and $b$, where the monomial $u_{S}=u_{1} \cdots u_{n}$ is given by

$$
u_{i}= \begin{cases}b & \text { if } i \in S, \\ a & \text { if } i \notin S .\end{cases}
$$

The $a b$-index of an Eulerian poset is then a polynomial of $c=a+b$ and $d=$ $a b+b a$. This polynomial $\Phi_{P}(c, d)$ is called the $c d$-index of $P$. As was observed by Stanley [19], the existence of the $c d$-index is equivalent to stating that the ce-index, obtained by rewriting the $a b$-index as a polynomial of $c=a+b$ and $e=a-b$, is a polynomial of $c$ and $e^{2}$. Let us denote by $L_{S}$ the coefficient of the ce-word $v_{1} \cdots v_{n}$ given by

$$
v_{i}= \begin{cases}e & \text { if } i \in S \\ c & \text { if } i \notin S\end{cases}
$$

in the $c e$-index. It was shown by Bayer and Hetyei [4] that the resulting flag $L$ vector $\left(L_{S}: S \subseteq[1, n]\right)$ of a graded poset of rank $n+1$ is connected to the flag $f$-vector by the formulas

$$
L_{S}=(-1)^{n-|S|} \sum_{T \supseteq[1, n] \backslash S}\left(-\frac{1}{2}\right)^{|T|} f_{T} \quad \text { and } \quad f_{S}=2^{|S|} \sum_{T \subseteq[1, n] \backslash S} L_{T} .
$$

The Bayer-Billera relations are thus also equivalent to stating that, for an Eulerian poset, $L_{S}=0$ unless $S$ is an even set, i.e., a disjoint union of intervals of even cardinality. A short direct proof of this equivalence may be found in [5].

The toric $h$-vector associated to a graded Eulerian poset $\widehat{P}=[\widehat{0}, \widehat{1}]$ was defined by Stanley [20] by introducing the polynomials $f([\widehat{0}, \widehat{1}), x)$ and $g([\widehat{0}, \widehat{1}), x)$ via the intertwined recurrences

$$
\begin{gathered}
f([\widehat{0}, \widehat{1}), x)=\sum_{p \in \widehat{0}, \widehat{1})} g([\widehat{0}, p), x)(x-1)^{\rho(\widehat{1})-1-\rho(p)} \quad \text { and } \\
g([\widehat{0}, \widehat{1}), x)=\sum_{i=0}^{\lfloor(\rho(\widehat{1})-1) / 2\rfloor}\left(\left[x^{i}\right] f([\widehat{0}, \widehat{1}), x)-\left[x^{i-1}\right] f([\widehat{0}, \widehat{1}), x)\right) x^{i}
\end{gathered}
$$

subject to the initial conditions $f(\emptyset, x)=g(\emptyset, x)=1$. Here the intervals $P=[\widehat{0}, \widehat{1})$ and $[\widehat{0}, p)$ are half open: they contain the minimum element $\widehat{0}$ but they do not contain their maximum element. In particular, $f(\emptyset, x)$ and $g(\emptyset, x)$ are associated 
to the only Eulerian poset of rank 0 . The toric $h$-vector associated to $[\widehat{0}, \widehat{1})$ is then defined as the vector of coefficients of the polynomial $f(\widehat{0}, \widehat{1}), x)$ :

$$
\sum_{i} h_{i} x^{i}:=x^{\rho(\widehat{1})-1} f([\widehat{0}, \widehat{1}), 1 / x) .
$$

By [20, Theorem 2.4], the polynomial above also equals $f([\widehat{0}, \widehat{1}), x)$; the apparently more complicated definition given by Stanley 20 is made for the sake of generalizations to lower Eulerian posets. The first formula expressing the polynomials $f([\widehat{0}, \widehat{1}), x)$ in terms of the flag $f$-vector was found by Fine (see [1] and [3. Theorem 7.14]). Here we state it in an equivalent form that appears in the paper of Bayer and Ehrenborg [3, Section 7]:

$$
f([\widehat{0}, \widehat{1}), x)=\sum_{S \subseteq[1, n]} f_{S} \sum_{\lambda \in\{-1,1\}^{n}: S(\lambda) \supseteq S}(-1)^{|S|+n-i_{\lambda}} x^{i_{\lambda}},
$$

where $S(\lambda)=\left\{s \in[1, n]: \lambda_{1}+\cdots+\lambda_{s}>0\right\}$ and $i_{\lambda}$ is the number of -1 's in $\lambda$. Bayer and Ehrenborg [3, Theorem 4.2] also expressed the toric $h$-vector of an Eulerian poset in terms of its $c d$-index.

\section{AdDitive AND MUltipliCATIVE SYMmetRy OF POLYNOMIALS}

Definition 2.1. Let $p(x) \in K[x]$ be a polynomial of degree $n$, with coefficients from a field $K$. We say that $p(x)$ is multiplicatively symmetric if it satisfies $x^{n} p\left(x^{-1}\right)=$ $p(x)$ and $p(x)$ is additively symmetric if it satisfies $p(x)=(-1)^{n} p(-x)$.

Lemma 2.2. A polynomial $p(x)=a_{n} x^{n}+a_{n-1} x^{n-1}+\cdots+a_{0}$ of degree $n$ is multiplicatively symmetric if and only if its coefficients satisfy $a_{k}=a_{n-k}$ for $0 \leq$ $k \leq n$.

Lemma 2.3. A polynomial $p(x)=a_{n} x^{n}+a_{n-1} x^{n-1}+\cdots+a_{0}$ of degree $n$ is additively symmetric if and only if its coefficients satisfy $a_{n-2 k-1}=0$ for $0 \leq k \leq$ $\lfloor(n-1) / 2\rfloor$.

Theorem 2.4. A polynomial $p(x) \in K[x]$ of degree $n$ is multiplicatively symmetric if and only if there is an additively symmetric polynomial $q(x) \in K[x]$ of degree $n$ satisfying

$$
p(x)=x^{\frac{n}{2}}\left(q(\sqrt{x})+q\left(\frac{1}{\sqrt{x}}\right)-q(0)\right) .
$$

Moreover, $q(x)$ is uniquely determined.

Proof. Assume first that $p(x)$ may be written in the form given in (6) using an additively symmetric polynomial $q(x)=\sum_{k=0}^{\lfloor n / 2\rfloor} a_{n-2 k} x^{n-2 k}$ of degree $n$. Then we have

$$
p(x)=\sum_{k=0}^{\left\lfloor\frac{n}{2}\right\rfloor} a_{n-2 k}\left(x^{n-k}+x^{k}\right)-x^{\frac{n}{2}} q(0) .
$$

For odd $n$ we have $q(0)=0$; thus $p(x)$ is a polynomial of degree $n$. Clearly, it is multiplicatively symmetric. 
Assume now that $p(x) \in K[x]$ is multiplicatively symmetric of degree $n$. If $n$ is even, then $p(x)$ is of the form

$$
\begin{aligned}
p(x) & =a_{\frac{n}{2}} x^{\frac{n}{2}}+\sum_{k=1}^{\frac{n}{2}} a_{\frac{n}{2}-k}\left(x^{\frac{n}{2}+k}+x^{\frac{n}{2}-k}\right) \\
& =x^{\frac{n}{2}}\left(a_{\frac{n}{2}}+\sum_{k=1}^{\frac{n}{2}} a_{\frac{n}{2}-k}\left((\sqrt{x})^{2 k}+(\sqrt{x})^{-2 k}\right)\right),
\end{aligned}
$$

and it satisfies (6) with $q(x)=a_{n / 2}+\sum_{k=1}^{n / 2} a_{n / 2-k} x^{2 k}$.

If $n$ is odd, then $p(x)$ is of the form

$$
\begin{aligned}
p(x) & =\sum_{k=0}^{\frac{n-1}{2}} a_{\frac{n-2 k-1}{2}}\left(x^{\frac{n-2 k-1}{2}}+x^{\frac{n+2 k+1}{2}}\right) \\
& =x^{\frac{n}{2}}\left(\sum_{k=0}^{\frac{n-1}{2}} a_{\frac{n-2 k-1}{2}}\left((\sqrt{x})^{2 k+2}+(\sqrt{x})^{-2 k-2}\right)\right),
\end{aligned}
$$

and it satisfies (6) with $q(x)=\sum_{k=0}^{(n-1) / 2} a_{(n-2 k-1) / 2} x^{2 k+1}$.

We are left to show that $q(x):=\sum_{k=0}^{\lfloor n / 2\rfloor} b_{k} x^{n-2 k}$ is uniquely determined by (6). For $k<n / 2, b_{k}$ must equal $\left[x^{n-k}\right] p(x)$. Finally, for even $n, b_{n / 2}$ must equal $\left[x^{n / 2}\right] p(x)$.

Definition 2.5. Given a multiplicatively symmetric polynomial $p(x)$ we call the additively symmetric variant of $p(x)$ the additively symmetric polynomial $q(x)$ associated to $p(x)$ via (6). Conversely, given an additively symmetric polynomial $q(x)$ we call the multiplicatively symmetric variant of $q(x)$ the polynomial $p(x)$ defined by (므).

It should be noted that the additively symmetric variant of a multiplicatively symmetric polynomial has the "same coefficients", without the redundant repetitions. For example, the additively symmetric variant of $p(x)=1-2 x^{2}-2 x^{5}+x^{7}$ is $q(x)=x^{7}-2 x^{3}$, and the additively symmetric variant of $p(x)=1+2 x+x^{2}$ is $q(x)=x^{2}+2$.

To express the additively symmetric variant of a multiplicatively symmetric polynomial, we may use the following truncation operators.

Definition 2.6. Let $K$ be a fixed field. For any $z \in \mathbb{Z}$, the truncation operator $U_{\geq z}: K\left[x, x^{-1}\right] \rightarrow K\left[x, x^{-1}\right]$ is the linear operator defined on the vector space $K\left[x, x^{-1}\right]$ of Laurent polynomials by discarding all terms of degree less than $z$. Similarly $U_{\leq z}: K\left[x, x^{-1}\right] \rightarrow K\left[x, x^{-1}\right]$ is the linear operator defined by discarding all terms of degree more than $z$.

The notation $U_{\geq z}$ and $U_{\leq z}$ is consistent with the notation used by Bayer and Ehrenborg [3], who rewrite (44) as

$$
g([\widehat{0}, \widehat{1}), x)=U_{\leq\lfloor n / 2\rfloor}((1-x) f([\widehat{0}, \widehat{1}), x)) .
$$

Lemma 2.7. Let $p(x)$ be a multiplicatively symmetric polynomial of degree $n$. Then the additively symmetric variant $q(x)$ of $p(x)$ satisfies

$$
q(x)=U_{\geq 0}\left(x^{-n} p\left(x^{2}\right)\right)=U_{\geq 0}\left(x^{n} p\left(x^{-2}\right)\right) .
$$


Proof. Substituting $x^{2}$ into (6) and dividing both sides by $x^{n}$ yields

$$
x^{-n} p\left(x^{2}\right)=q(x)+q\left(\frac{1}{x}\right)-q(0) .
$$

Applying $U_{\geq 0}$ to both sides results in $q(x)$ on the right hand side. Finally, $x^{-n} p\left(x^{2}\right)$ $=x^{n} p\left(x^{-2}\right)$ is an immediate consequence of the multiplicative symmetry of $p(x)$.

\section{The SHORT TORIC POLYNOMIAL OF AN ARBITRARY GRADED POSET}

Stanley's generalization [20, Theorem 2.4] of the Dehn-Sommerville equations to the toric polynomial $f([\widehat{0}, \widehat{1}), x)$ associated to an Eulerian poset $[\widehat{0}, \widehat{1}]$ states the following.

Theorem 3.1 (Stanley). For an Eulerian poset $[\widehat{0}, \widehat{1}]$ of rank $n+1$, the polynomial $f(\widehat{0}, \widehat{1}), x)$ is multiplicatively symmetric of degree $n$.

Definition 3.2. The short toric polynomial $\mathrm{t}([\widehat{0}, \widehat{1}), x)$ associated to an Eulerian poset $[\widehat{0}, \widehat{1}]$ is the additively symmetric variant of the toric polynomial $f([\widehat{0}, \widehat{1}), x)$.

As in the case of $f([\widehat{0}, \widehat{1}), x)$, we consider the interval $[\widehat{0}, \widehat{1})$ to be half open. In particular, $f(\emptyset, x)=1$ is restated as $\mathrm{t}(\emptyset, x)=1$. As an immediate consequence of Definitions 2.5 and 3.2 we obtain that any Eulerian poset $[\widehat{0}, \widehat{1}]$ of rank $n+1$ satisfies

$$
f([\widehat{0}, \widehat{1}), x)=x^{\frac{n}{2}}\left(\mathrm{t}([\widehat{0}, \widehat{1}), \sqrt{x})+\mathrm{t}\left([\widehat{0}, \widehat{1}), \frac{1}{\sqrt{x}}\right)-\mathrm{t}([\widehat{0}, \widehat{1}), 0)\right) .
$$

Furthermore, by Lemma 2.7, we have

$$
\left.\mathrm{t}((\widehat{0}, \widehat{1}), x)=U_{\geq 0}\left(x^{-n} f(\widehat{0}, \widehat{1}), x^{2}\right)\right)=U_{\geq 0}\left(x^{n} f\left((\widehat{0}, \widehat{1}), x^{-2}\right)\right) .
$$

Lemma 3.3. If $[\widehat{0}, \widehat{1}]$ is an Eulerian poset of rank $n+1$, then we have

$$
U_{\geq 1}\left(\mathrm{t}([\widehat{0}, \widehat{1}), x) \cdot\left(x-\frac{1}{x}\right)\right)=x^{n+1} g\left([\widehat{0}, \widehat{1}), x^{-2}\right) .
$$

Proof. After substituting $x^{-2}$ into (7), multiplying both sides by $x^{n+1}$, and using the obvious identity $x^{n+1} U_{\geq-n}(p(x))=U_{\geq 1}\left(x^{n+1} p(x)\right)$ we obtain

$$
x^{n+1} g\left((\widehat{0}, \widehat{1}), x^{-2}\right)=U_{\geq 1}\left(\left(x-x^{-1}\right) x^{n} f\left([\widehat{0}, \widehat{1}), x^{-2}\right)\right) .
$$

Only terms of degree at least 0 in $x^{n} f\left([\widehat{0}, \widehat{1}), x^{-2}\right)$ yield a term of degree at least 1 in $\left(x-x^{-1}\right) x^{n} f\left([\widehat{0}, \widehat{1}), x^{-2}\right)$. Thus we may also write

$$
x^{n+1} g\left([\widehat{0}, \widehat{1}), x^{-2}\right)=U_{\geq 1}\left(\left(x-x^{-1}\right) U_{\geq 0}\left(x^{n} f\left([\widehat{0}, \widehat{1}), x^{-2}\right)\right)\right),
$$

and the statement follows from (9).

Using (9) and Lemma 3.3 we may show the following fundamental recurrence for the short toric polynomial. 
Theorem 3.4. The short toric polynomial satisfies the recurrence

$$
\begin{aligned}
\mathrm{t}([\widehat{0}, \widehat{1}), x)=U_{\geq 0} & \left(x^{-1}-x\right)^{\rho(\widehat{1})-1} \\
& \left.+\sum_{\widehat{0}<p<\widehat{1}} U_{\geq 1}\left(\mathrm{t}([\widehat{0}, p), x)\left(x-x^{-1}\right)\right)\left(x^{-1}-x\right)^{\rho(\widehat{1})-\rho(p)-1}\right) .
\end{aligned}
$$

Proof. Let us set $n:=\rho(\widehat{1})-1$. Substituting $x^{-2}$ into (33) and multiplying both sides by $x^{n}$ yields

$$
x^{n} f\left([\widehat{0}, \widehat{1}), x^{-2}\right)=\sum_{p \in \widehat{0}, \widehat{1})} x^{\rho(p)} g\left([\widehat{0}, p), x^{-2}\right)\left(x^{-1}-x\right)^{\rho(\widehat{1})-1-\rho(p)} .
$$

By (9), applying $U_{\geq 0}$ to the left hand side yields $\mathrm{t}([\widehat{0}, \widehat{1}), x)$. We only need to show that the right hand side of (10) is the argument of the operator $U_{\geq 0}$ on the right hand side of the statement. Since $g(\emptyset, x)=1$, the term associated to $p=\widehat{0}$ is $\left(x^{-1}-x\right)^{n}$ on the right hand side of (10). For $p \in(\widehat{0}, \widehat{1})$, the term $x^{\rho(p)} g\left([0, p), x^{-2}\right)$ equals $U_{\geq 1}\left(\mathrm{t}([\widehat{0}, p), x) \cdot\left(x-x^{-1}\right)\right)$ by Lemma $\underline{3.3}$.

Definition 3.5. We extend the definition of $\mathrm{t}([\widehat{0}, \widehat{1}), x)$ to all finite posets $P$ that have a unique minimum element $\widehat{0}$ and a rank function $\rho: P \rightarrow \mathbb{N}$, satisfying $\rho(\widehat{0})=0$, as follows. We set $\mathrm{t}(\emptyset, x):=1$ and

$$
\mathrm{t}(P, x)=U_{\geq 0}\left(\left(x^{-1}-x\right)^{n}+\sum_{p \in P \backslash\{\widehat{0}\}} U_{\geq 1}\left(\mathrm{t}([\widehat{0}, p), x)\left(x-x^{-1}\right)\right)\left(x^{-1}-x\right)^{n-\rho(p)}\right) .
$$

Here $n=\max \{\rho(p): p \in P\}$.

Stanley [20, §4] calls a finite poset lower Eulerian if it has a unique minimum element $\widehat{0}$ and, for each $p \in P$, the interval $[\widehat{0}, p]$ is an Eulerian poset. He extends the definition of the toric polynomial $f(P, x)$ to lower Eulerian posets by setting

$$
f(P, x)=\sum_{p \in P} g([\widehat{0}, p), x)(x-1)^{n-\rho(p)} .
$$

Here $n$ is the length of the longest chain in $P$.

Proposition 3.6. Let $P$ be a lower Eulerian poset and let $n$ be the length of the longest chain in $P$. Then we have

$$
\mathrm{t}(P, x)=U_{\geq 0}\left(x^{n} f\left(P, x^{-2}\right)\right) .
$$

Indeed, when we use the recurrence given in Definition 3.5 to compute $\mathrm{t}(P, x)$, all intervals $[\widehat{0}, p)$ on the right hand side are Eulerian posets with their maximum element removed. Thus we may repeat the part of the proof of Theorem 3.4 showing that the right hand side is $U_{\geq 0}\left(x^{n} f\left(P, x^{-2}\right)\right)$.

Remark 3.7. Unlike in the Eulerian case, the short toric polynomial $\mathrm{t}(P, x)$ of a lower Eulerian poset $P$ may not contain sufficient information to recover $f(P, x)$. Consider for example the case when $P=[\widehat{0}, \widehat{1}]$ is a graded Eulerian poset of rank $n+1$. Then, by [20, (19)],

$$
f([\widehat{0}, \widehat{1}], x)=x^{n+1} g([\widehat{0}, \widehat{1}), 1 / x)
$$


contains only terms of degree greater than $\lfloor n / 2\rfloor$. Thus, by Proposition [3.6, we obtain $\mathrm{t}([\widehat{0}, \widehat{1}], x)=0$.

Next we prove a generalization of the short toric variant of Fine's formula (5) to all posets $P$ for which $\mathrm{t}(P, x)$ is defined. Let $P$ be such a poset and let $n=$ $\max \{\rho(p): p \in P\}$. As for graded posets, for any $S \subseteq[1, n]$, we may define the number $f_{S}=f_{S}(P)$ as the number of maximal chains in the $S$ rank-selected subposet $P_{S}=\{u \in P: \rho(u) \in S\}$.

Proposition 3.8 (Fine's formula).

$$
\mathrm{t}(P, x)=\sum_{S \subseteq[1, n]} f_{S}(P) \cdot \sum_{\lambda \in\{-1,1\}^{n}: S(\lambda) \supseteq S, n-2 i_{\lambda} \geq 0}(-1)^{n-i_{\lambda}+|S|} x^{n-2 i_{\lambda}}
$$

holds for all finite posets $P$ having a unique minimum element $\widehat{0}$ and a rank function $\rho: P \rightarrow \mathbb{N}$, satisfying $\rho(\widehat{0})=0$ and $n=\max \{\rho(p): p \in P\}$.

Proof. The statement may be shown in a very similar fashion to Fine's original formula, the only difference being that the role of the intertwined recurrence equations (3) and (4) is taken over by the single recurrence given in Definition 3.5. making the proof somewhat simpler. Thus we only outline the proof. Introducing

$$
\mathrm{t}_{f}(S, n, x):=\sum_{\lambda \in\{-1,1\}^{n}: S(\lambda) \supseteq S, n-2 i_{\lambda} \geq 0}(-1)^{n-i_{\lambda}+|S|} x^{n-2 i_{\lambda}},
$$

we need to show $\mathrm{t}(P, x)=\sum_{S \subseteq[1, n]} f_{S}(P) \cdot \mathrm{t}_{f}(S, n, x)$. Observe first that the condition $n-2 i_{\lambda} \geq 0$ in the summation defining $\mathrm{t}_{f}(S, n, x)$ is equivalent to $\lambda_{1}+$ $\cdots+\lambda_{n} \geq 0$, and it may be dropped if we apply the operator $U_{\geq 0}$ instead. In particular, we have

$$
\left.\mathrm{t}_{f}(\emptyset, n, x)=U_{\geq 0}\left(\left(x^{-1}-x\right)^{n}\right)\right) ;
$$

thus, by Definition 3.5, we only need to prove

$$
\begin{aligned}
U_{\geq 0}\left(\sum_{p \in P \backslash\{0\}} U_{\geq 1}\left(\mathrm{t}([\widehat{0}, p), x)\left(x-x^{-1}\right)\right)\right. & \left.\left(x^{-1}-x\right)^{n-\rho(p)}\right) \\
& =\sum_{\emptyset \neq S \subseteq[1, n]} f_{S}(P) \cdot \mathrm{t}_{f}(S, n, x) .
\end{aligned}
$$

This statement may easily be shown by induction on $n$, using the fact that, for $S \neq \emptyset$, we have

$$
\left.\mathrm{t}_{f}(S, n, x)=U_{\geq 0}\left(U_{\geq 1}\left(\mathrm{t}_{f}(S \backslash\{\max S\}, \max S, x)\left(x-x^{-1}\right)\right)\left(x^{-1}-x\right)^{n-\max S}\right)\right)
$$

and

$$
f_{S}(P)=\sum_{p \in P: \rho(p)=\max S} f_{S \backslash\{\max S\}}([\widehat{0}, p]) .
$$

Corollary 3.9. The degree of $\mathrm{t}(P, x)$ equals $\max \{\rho(p): p \in P\}$ if and only if

$$
\sum_{S \subseteq[1, n]}(-1)^{|S|} f_{S}(P) \neq 0 .
$$


It is worth noting that $\sum_{S \subseteq[1, n]}(-1)^{|S|} f_{S}(P)$ is the reduced Euler characteristic of the order complex of $P \backslash\{\widehat{0}\}$ : this is the simplicial complex, whose vertex set is $P \backslash\{\widehat{0}\}$ and whose faces are the chains in $P \backslash\{\widehat{0}\}$. It is not difficult to show by induction on $n$, using Definition 3.5, that $\mathrm{t}(P, x)$ is additively symmetric for every $P$. However, in light of Proposition 3.6 and Remark 3.7, it makes more sense to call the coefficients of the multiplicatively symmetric variant of $\mathrm{t}(P, x)$ the "toric $h$-vector of $P$ " when $\mathrm{t}(P, x)$ has "full degree". According to Corollary [3.9] this is possible exactly when the reduced Euler characteristic of the order complex of $P \backslash\{\widehat{0}\}$ is not zero.

As was the case with Fine's formula and Eulerian posets, the short toric variant of Fine's formula above may be used to express $\mathrm{t}(P, x)$ in terms of the flag $h$ vector, whenever $\mathrm{t}(P, x)$ is defined. For that purpose we may directly adapt the ideas present in the work of Bayer and Ehrenborg [3, Section 7.4]. An outline may be found in the Appendix.

\section{The SHORT TORIC POLYNOMIAL AND THE $c d$-INDEX}

In this section we show how easy it is to compute the polynomial $\mathrm{t}([\widehat{0}, \widehat{1}), x)$, associated to an Eulerian poset $[\widehat{0}, \widehat{1}]$, from its $c d$-index. We begin by expressing $\mathrm{t}([\widehat{0}, \widehat{1}), x)$ in terms of the $c e$-index.

Using (2) and the binomial theorem we may rewrite (12) as

$$
\mathrm{t}([\widehat{0}, \widehat{1}), x)=\sum_{S \subseteq[1, n]} L_{S} \sum_{\lambda \in\{-1,1\}^{n}: n-2 i_{\lambda} \geq 0} x^{n-2 i_{\lambda}}(-1)^{n-i_{\lambda}+|S(\lambda) \backslash S|} .
$$

Just as in [3, Section 7.4], we represent each $\lambda \in\{-1,1\}^{n}$ by a lattice path $\Lambda(\lambda)$ starting at $(0,0)$ and containing $\left(1, \lambda_{i}\right)$ as step $i$ for $i=1, \ldots, n$. The resulting lattice path has $n$ steps, each step is a northeast step $(1,1)$ or a southeast step $(1,-1)$. The condition $n-2 i_{\lambda} \geq 0$ is equivalent to stating that we only consider lattice paths whose right endpoint is on or above the horizontal axis. As is usual in lattice-path combinatorics, we may use the "reflection principle" to match canceling terms, thus simplifying the summation. For that purpose, let us introduce $R(\lambda):=$ $\left\{i \in[1, n]: \lambda_{1}+\cdots+\lambda_{i}=0\right\}$. Note that $R(\lambda)$ necessarily consists of even integers. We also say that a set $S$ evenly contains the set $R$ if $R \subseteq S$ and $S \backslash R$ is the disjoint union of intervals of even cardinality.

Proposition 4.1. Let $[\widehat{0}, \widehat{1}]$ be a graded Eulerian poset of rank $n+1$. Then we have

$$
\mathrm{t}([\widehat{0}, \widehat{1}), x)=\sum_{S \subseteq[1, n]} L_{S} \cdot \mathrm{t}_{c e}(S, n, x) .
$$

Here $\mathrm{t}_{c e}(S, n, x)$ is the total weight of all $\lambda \in\{-1,1\}^{n}$ such that $S$ evenly contains $R(\lambda) \cup(R(\lambda)-1)$ and $\lambda_{1}+\cdots+\lambda_{n} \geq 0$. The weights are defined as follows:

(1) each $i \in[1, n]$ such that $\lambda_{i}=-1$ contributes a factor of $1 / x$;

(2) each $i \in[1, n]$ such that $\lambda_{i}=1$ contributes a factor of $-x$;

(3) each $i \in S(\lambda) \backslash S$ contributes an additional factor of -1 .

Proof. If we remove the condition that $S$ evenly contains $R(\lambda) \cup(R(\lambda)-1)$, we obtain an exact rephrasing of (13). We only need to show that the vectors $\lambda \in\{-1,1\}^{n}$ such that $S$ does not contain $R(\lambda) \cup(R(\lambda)-1)$ evenly may be matched into pairs whose weights cancel. 
Since $R(\lambda):=\left\{r_{1}, \ldots, r_{k}\right\}$ consists of even integers only, the set $R(\lambda) \uplus(R(\lambda)-1)$ may be written as the disjoint union

$$
R(\lambda) \cup(R(\lambda)-1)=\biguplus_{i=1}^{k}\left[r_{i}-1, r_{i}\right] .
$$

We may assume that $S$ is an even set; otherwise $L_{S}=0$. Then $S:=\left\{s_{1}, s_{2}, \ldots, s_{2 m}\right\}$ may be written as

$$
S=\biguplus_{j=1}^{m}\left[s_{2 j-1}, s_{2 j-1}+1\right] .
$$

It is easy to see that $R(\lambda) \cup(R(\lambda)-1)$ is evenly contained in $S$ if and only if each $\left[r_{i}-1, r_{i}\right]$ appearing on the right hand side of (14) equals some $\left[s_{2 j-1}, s_{2 j-1}+1\right]$ on the right hand side of (15). Assume $S$ does not contain $R(\lambda) \cup(R(\lambda)-1)$ evenly and let $i$ be the least index such that $\left[r_{i}-1, r_{i}\right]$ does not equal any $\left[s_{2 j-1}, s_{2 j-1}+1\right]$. Then either $r_{i}-1 \notin S$ or $r_{i}-1$ is the right end of some interval $\left[s_{2 j-1}, s_{2 j-1}+1\right]$. Either way $\left|S \cap\left[1, r_{i}-1\right]\right|$ is even. After setting $r_{0}:=0$, we may also state that $S \cap\left[1, r_{i-1}\right]$ has even cardinality: it is the empty set when $i=1$, and it is the disjoint union of some intervals $\left[s_{2 j-1}, s_{2 j-1}+1\right]$ when $i>1$. Combining the previous two observations, we obtain that $\left|S \cap\left[r_{i-1}+1, r_{i}-1\right]\right|$ is even. On the other hand, $\left|\left[r_{i-1}+1, r_{i}-1\right]\right|$ is odd since $r_{i-1}$ and $r_{i}$ are even. Therefore $\left|\left[r_{i-1}+1, r_{i}-1\right] \backslash S\right|$ is odd. Now consider the vector $\tilde{\lambda}$ that corresponds to the lattice path obtained by reflecting the part of the lattice path associated to $\lambda$ between $\left(r_{i-1}, 0\right)$ and $\left(r_{i}, 0\right)$. The reflected part has the same number of northeast steps and southeast steps; thus, up to sign, $\lambda$ and $\tilde{\lambda}$ have the same weight. We also have $R(\lambda)=R(\tilde{\lambda})$ and $\tilde{\tilde{\lambda}}=\lambda$. Finally, because $\left|\left[r_{i-1}+1, r_{i}-1\right] \backslash S\right|$ is odd, item (3) above implies that the weights of $\lambda$ and $\tilde{\lambda}$ cancel.

We may use the "reflection principle" to compute the polynomials $\mathrm{t}_{c e}(S, n, x)$ defined in Proposition 4.1 even more efficiently, as the total weight of even fewer vectors $\lambda \in\{1,1\}^{n}$.

Theorem 4.2. Using a new weighting, for any $n \geq 1$ and any $S \subseteq[1, n]$, the polynomial $\mathrm{t}_{c e}(S, n, x)$ also equals the total weight of all $\lambda \in\{-1,1\}^{n}$ such that $S$ evenly contains $R(\lambda) \cup(R(\lambda)-1)$ and $\lambda_{1}+\cdots+\lambda_{i} \geq 0$ holds for all $i \in[1, n]$. The new weight of each $\lambda$, satisfying the conditions stated above, is defined as follows:

(1) each $\lambda_{i}=-1$ contributes a factor of $-1 / x$;

(2) each $\lambda_{i}=1$ contributes a factor of $x$;

(3) each element of $R(\lambda)$ contributes an additional factor of 2 .

Proof. Consider the way described in Proposition 4.1 to compute the polynomials $\mathrm{t}_{c e}(S, n, x)$ as the total weight of all $\lambda \in\{-1,1\}^{n}$. Let us say that $\nu \in\{-1,1\}^{n}$ is a reflection of $\lambda$ if there is an $r_{i} \in R(\lambda)=\left\{r_{1}, \ldots, r_{k}\right\}$ such that the lattice path $\Lambda(\nu)$ corresponding to $\nu$ is obtained from the lattice path $\Lambda(\lambda)$ corresponding to $\lambda$ by reflecting the part of $\Lambda(\lambda)$ between $\left(r_{i-1}, 0\right)$ and $\left(r_{i}, 0\right)$. (As before, we set $r_{0}:=0$.) An argument completely analogous to the proof of Proposition 4.1 above shows that $\lambda$ has the same weight as $\nu$ with respect to the weighting defined there. Consider the equivalence relation obtained by taking the transitive closure of the relation " $\nu$ is a reflection of $\lambda$ ". Clearly, the number of elements in the equivalence class of $\lambda$ is $2^{|R(\lambda)|}$ and $\lambda$ being equivalent to $\nu$ implies $R(\lambda)=R(\nu)$ and $\lambda$ and $\nu$ have the same 
weight. Thus we may replace each $\lambda$ with the only vector $\lambda^{+}$in its equivalence class satisfying $\lambda_{1}^{+}+\cdots+\lambda_{i}^{+} \geq 0$ for all $i \in\left[1, \max \left(R\left(\lambda^{+}\right)\right)\right]$, at the expense of introducing an additional factor of 2 , contributed by each $i \in R\left(\lambda^{+}\right)$. Obviously, each such class representative $\lambda^{+}$exists uniquely. Observe next that each class representative $\lambda^{+}$ satisfies $\lambda_{1}^{+}+\cdots+\lambda_{i}^{+} \geq 0$ for all $i$. This follows from $\lambda_{1}^{+}+\cdots+\lambda_{n}^{+} \geq 0$ and from the fact that the associated lattice path of $\lambda^{+}$cannot cross the horizontal axis after having reached the point $\left(\max \left(R\left(\lambda^{+}\right)\right), 0\right)$. Thus we have $S\left(\lambda^{+}\right)=[1, n] \backslash R\left(\lambda^{+}\right)$. Since $S$ contains $R\left(\lambda^{+}\right)$, the set $S\left(\lambda^{+}\right)=[1, n] \backslash R\left(\lambda^{+}\right)$contains $[1, n] \backslash S$ and $S\left(\lambda^{+}\right) \backslash S=[1, n] \backslash S$. Thus, for each $\lambda^{+}$, item (3) in Proposition 4.1 is equivalent to requiring that each $i \in[1, n] \backslash S$ contributes an additional factor of -1 . Since $S$ is an even set, $|[1, n] \backslash S|$ has the same parity as $n$. Therefore, for the class representatives $\lambda^{+}$, we may remove item (3) in Proposition 4.1 after changing the signs of the contributions in items (1) and (2).

Theorem 4.2 gains an even simpler form when we rephrase it in terms of the $c d$-index.

Theorem 4.3. Let $[\widehat{0}, \widehat{1}]$ be a graded Eulerian poset of rank $n+1$. Then we have

$$
\mathrm{t}([\widehat{0}, \widehat{1}), x)=\sum_{w}[w] \Phi_{[\widehat{0}, \widehat{1}]}(c, d) \cdot \mathrm{t}(w, x) .
$$

Here the summation runs over all cd-words $w$ of degree $n$. The polynomial $\mathrm{t}(w, x)$ is the total weight of all $\lambda \in\{-1,1\}^{n}$ such that the set of positions covered by letters $d$ equals $R(\lambda) \cup(R(\lambda)-1)$ and $\lambda_{1}+\cdots+\lambda_{i} \geq 0$ holds for all $i \in[1, n]$. The weight of each such $\lambda$ is defined as follows:

(1) each $\lambda_{i}=-1$ contributes a factor of $-1 / x$;

(2) each $\lambda_{i}=1$ contributes a factor of $x$;

(3) each element of $R(\lambda)$ contributes an additional factor of -1 .

Proof. Let us use the relation $d=\frac{1}{2} \cdot\left(c^{2}-e^{2}\right)$ to express each $c d$-word $w$ as a linear combination

$$
w=\sum_{w^{\prime}} \alpha_{w, w^{\prime}} w^{\prime}
$$

of $c e$-words $w^{\prime}$, and for each $c e$-word $w^{\prime}$ let us denote by $S\left(w^{\prime}\right)$ the set of positions covered by a letter $e$ in $w^{\prime}$. (The coefficient of $w^{\prime}$ in the ce-index is thus $L_{S\left(w^{\prime}\right)}$.) By Proposition 4.1 and by Theorem 4.2, it suffices to show that the polynomial $\mathrm{t}(w, x)$ given in our statement satisfies

$$
\mathrm{t}(w, x)=\sum_{w^{\prime}} \alpha_{w, w^{\prime}} \mathrm{t}_{c e}\left(S\left(w^{\prime}\right), n, x\right) .
$$

Using the weighting introduced in Theorem 4.2, a vector $\lambda \in\{-1,1\}^{n}$ contributes to some $\mathrm{t}_{c e}\left(S\left(w^{\prime}\right), n, x\right)$ only if the $S\left(w^{\prime}\right)$ evenly contains $R(\lambda) \cup(R(\lambda)-1)$. Since the factors $e^{2}$ only arise from expanding $d \mathrm{~s}$, if $\lambda$ contributes to $\mathrm{t}(w, x)$ for some $c d-$ word $w$, the set of positions covered by $d$ must also evenly contain $R(\lambda) \cup(R(\lambda)-1)$. We claim that, in order to have a nonzero contribution, the two sets must be equal. Assume, by way of contradiction, that the set of positions covered by $d$ 's in $w$ properly contains $R(\lambda) \cup(R(\lambda)-1)$. Due to the requirement of even containment, this implies that there is a letter $d$ of $w$, covering the positions $k$ and $k+1$, such that the set $\{k, k+1\}$ is disjoint from $R(\lambda) \cup(R(\lambda)-1)$. Consider the $c e$-words appearing in the expansion (16) of $w$. These all have the property that the positions 
$k$ and $k+1$ are covered by either $c^{2}$ or $e^{2}$ and the map $w^{\prime} \mapsto \iota\left(w^{\prime}\right)$ replacing $c^{2}$ with $e^{2}$ and vice versa in these positions is an involution. Our vector $\lambda$ contributes a nonzero weight to $\mathrm{t}_{c e}\left(S\left(w^{\prime}\right), n, x\right)$ if and only if it contributes a nonzero weight to $\mathrm{t}_{c e}\left(S\left(\iota\left(w^{\prime}\right)\right), n, x\right)$. Whenever this happens, the two contributions cancel.

We are left with considering vectors $\lambda \in\{-1,1\}^{n}$ such that the set of positions covered by $d$ 's in $w$ equals $R(\lambda) \cup(R(\lambda)-1)$. For these, only the ce-word $w^{\prime}$ obtained from $w$ by replacing every $d$ with $-1 / 2 \cdot e^{2}$ has the property that the positions covered by $e$ 's evenly contain $R(\lambda) \cup(R(\lambda)-1)$. For this $w^{\prime}$ we have $\alpha_{w, w^{\prime}}=(-1 / 2)^{|R(\lambda)|}$. The contribution of $\lambda$ to $\mathrm{t}(w, x)$ may be described by the weighting above, considering that the factors of 2 , contributed by the elements of $R(\lambda)$ to $\mathrm{t}_{c e}\left(S\left(w^{\prime}\right), n, x\right)$, multiplied with $\alpha_{w, w^{\prime}}$ leave us with a factor of -1 for each element of $R(\lambda)$.

Theorem 4.3 allows us to explicitly compute the contribution $\mathrm{t}(w, x)$. Thus we obtain the short toric equivalent of the result by Bayer and Ehrenborg [3, Theorem $4.2]$, expressing $f([\widehat{0}, \widehat{1}), x)$ in terms of the $c d$-index.

Proposition 4.4. The polynomial $\mathrm{t}\left(c^{k_{1}} d c^{k_{2}} \cdots c^{k_{r}} d c^{k}, x\right)$ is zero if at least one of $k_{1}, k_{2}, \ldots, k_{r}$ is odd. If $k_{1}, k_{2}, \ldots, k_{r}$ are all even, then

$$
\mathrm{t}\left(c^{k_{1}} d c^{k_{2}} \cdots c^{k_{r}} d c^{k}, x\right)=(-1)^{\frac{k_{1}+\cdots+k_{r}}{2}} C_{\frac{k_{1}}{2}} \cdots C_{\frac{k_{r}}{2}} \widetilde{Q}_{k}(x) .
$$

Here $C_{k}=\frac{1}{k+1}\left(\begin{array}{c}2 k \\ k\end{array}\right)$ is a Catalan number, and the polynomials $\widetilde{Q}_{n}(x)$ are given by $\widetilde{Q}_{0}(x)=1$ and

$$
\widetilde{Q}_{n}(x):=\sum_{j=0}^{\left\lfloor\frac{n-1}{2}\right\rfloor}(-1)^{j}\left(\left(\begin{array}{c}
n-1 \\
j
\end{array}\right)-\left(\begin{array}{c}
n-1 \\
j-1
\end{array}\right)\right) x^{n-2 j} \quad \text { for } n \geq 1 .
$$

Proof. To calculate $\mathrm{t}\left(c^{k_{1}} d c^{k_{2}} \cdots c^{k_{r}} d c^{k_{r+1}}, x\right)$ we must sum the weight of all vectors $\lambda \in\{-1,1\}^{n}$ whose associated lattice path is staying weakly above the horizontal axis, touching it exactly at the points $\left(k_{1}+2,0\right),\left(k_{1}+k_{2}+4,0\right), \ldots,\left(k_{1}+\cdots+\right.$ $\left.k_{r}+2 r, 0\right)$. There is no such lattice path if at least one of $k_{1}, k_{2}, \ldots, k_{r}$ is odd. Otherwise, we may select the lattice path by independently selecting Dyck paths of length $k_{1}+2, k_{2}+2, \ldots, k_{r}+2$ such that none of these Dyck paths touches the horizontal axis between its start and end, and then we may independently select a lattice path of length $k$ that stays strictly above the horizontal axis. It is immediate from the definition of the weighting that a lattice path that ends at $(n, j)$ contributes a term $\pm x^{j}$. The total weight of the Dyck paths is

$$
(-1)^{\frac{k_{1}+\cdots+k_{r}}{2}} C_{\frac{k_{1}}{2}} \cdots C_{\frac{k_{r}}{2}}
$$

this needs to be multiplied by the total weight of all lattice paths of length $k$ staying strictly above the horizontal axis. It is easy to verify that this weight is $\widetilde{Q}_{k}(x)$.

Remark 4.5. The polynomials $\widetilde{Q}_{n}(x)$ are closely related to the polynomials $Q_{n}(x)$ introduced by Bayer and Ehrenborg [3]. They may be given by the formula

$$
\widetilde{Q}_{n}(x)=x^{n} Q_{n}\left(x^{-2}\right) .
$$


In analogy to the results thus far in this section, it is possible to express $\mathrm{t}(P, x)$ of an arbitrary finite poset $P$ having a unique minimum element $\widehat{0}$ and a rank function $\rho: P \rightarrow \mathbb{N}$, in terms of its flag $h$-vector. This may be found in the Appendix.

Besides yielding the short toric analogue of [3, Theorem 4.2], Theorem 4.3 allows us to introduce two linear maps $\mathcal{C}: \mathbb{Q}[x] \rightarrow \mathbb{Q}[x]$ and $\mathcal{D}: \mathbb{Q}[x] \rightarrow \mathbb{Q}[x]$ in such a way that, for any graded Eulerian poset $[\widehat{0}, \widehat{1}]$, the polynomial $\mathrm{t}([\widehat{0}, \widehat{1}), x)$ may be computed by substituting $\mathcal{C}$ into $c$ and $\mathcal{D}$ into $d$ in the reverse of $\Phi_{P}(c, d)$ and applying the resulting linear operator to 1 . Note that the definitions and the result below are independent of the rank of $P$.

Definition 4.6. We define $\mathcal{C}: \mathbb{Q}[x] \rightarrow \mathbb{Q}[x]$ by

$$
\mathcal{C}\left(x^{n}\right)= \begin{cases}x^{n+1}-x^{n-1} & \text { if } n \geq 2 \\ x^{n+1} & \text { if } n \in\{0,1\}\end{cases}
$$

and $\mathcal{D}: \mathbb{Q}[x] \rightarrow \mathbb{Q}[x]$ by

$$
\mathcal{D}\left(x^{n}\right)= \begin{cases}-1 & \text { if } n=2 \\ 1 & \text { if } n=0 \\ 0 & \text { otherwise }\end{cases}
$$

Theorem 4.7. For any Eulerian poset $P=[\widehat{0}, \widehat{1}]$ we have

$$
\mathrm{t}([\widehat{0}, \widehat{1}), x)=\Phi_{P}^{\mathrm{rev}}(\mathcal{C}, \mathcal{D})(1) .
$$

Here $\Phi_{P}^{\mathrm{rev}}(\mathcal{C}, \mathcal{D})$ is obtained from $\Phi_{P}(c, d)$ by first taking the reverse of each $c d$ monomial and then replacing each $c$ with $\mathcal{C}$ and each $d$ with $\mathcal{D}$.

Proof. By Theorem 4.3, we only need to show that

$$
\mathrm{t}\left(c^{k_{1}} d c^{k_{2}} \cdots c^{k_{r}} d c^{k}, x\right)=\mathcal{C}^{k} \mathcal{D} \mathcal{C}^{k_{r}} \mathcal{D} \mathcal{C}^{k_{r-1}} \mathcal{D} \cdots \mathcal{D} \mathcal{C}^{k_{1}}(1)
$$

holds for any $c d$-word $w=c^{k_{1}} d c^{k_{2}} \cdots c^{k_{r}} d c^{k}$. This may be shown by induction on the degree of $w$, the basis being $\mathrm{t}(\varepsilon, t)=1$, where $\varepsilon$ is the empty word. Assume now that (18) holds for some $c d$-word $w$ of degree $n$ above. By Theorem 4.3 , each $\lambda \in\{-1,1\}^{n}$, contributing to $\mathrm{t}(w, x)$ corresponds to a lattice path remaining weakly above the horizontal axis such that each lattice point $(j, 0)$ on the lattice path satisfies that the positions $j$ and $j-1$ are covered by the same letter $d$ in $w$. The weight contributed by $\lambda$ is $\pm x^{\lambda_{1}+\cdots+\lambda_{n}}$, where $\lambda_{1}+\cdots+\lambda_{n}$ is the height of the right end of the corresponding lattice path. To compute $\mathrm{t}(w c, x)$, we must continue each lattice path contributing to $\mathrm{t}(w, x)$ with an additional step in such a way that the resulting lattice path must end strictly above the horizontal axis, and to multiply the weight of the lattice path with the weight of the additional step. This amounts to applying $\mathcal{C}$ to $\mathrm{t}(w, x)$, yielding

$$
\mathrm{t}(w c, x)=\mathcal{C} \mathrm{t}(w, x) .
$$

Similarly, to compute $\mathrm{t}(w d, x)$ we must continue each lattice path contributing to $\mathrm{t}(w, x)$ with two steps in such a way that the resulting lattice path ends on the horizontal axis. Clearly this can be done to the lattice paths of positive length and of weight $\pm x^{2}$ or 1 only. This amounts to applying $\mathcal{D}$ to $\mathrm{t}(w, x)$, yielding

$$
\mathrm{t}(w d, x)=\mathcal{D} \mathrm{t}(w, x) .
$$




\section{Two useful Bases}

Proposition 4.4 highlights the importance of the basis $\left\{\widetilde{Q}_{n}(x)\right\}_{n \geq 0}$ of the vector space $\mathbb{Q}[x]$. In this section we express the elements of the basis $\left\{x^{n}\right\}_{n \geq 0}$, as well as the operators $\mathcal{C}$ and $\mathcal{D}$, in this new basis. We also find the analogous results for the basis $\left\{t_{n}(x)\right\}_{n \geq 0}$, where $t_{n}(x)$ is defined as $\mathrm{t}\left(B_{n+1}, x\right)$ for the Boolean algebra $\widehat{B}_{n+1}$ of rank $n+1$. This basis will be useful in proving the main result of Section 6 , as well as in finding a very simple formula connecting $\mathrm{t}(P, x)$ with $g(P, x)$. Both $\left\{\widetilde{Q}_{n}(x)\right\}_{n \geq 0}$ and $\left\{t_{n}(x)\right\}_{n \geq 0}$ are bases of $\mathbb{Q}[x]$ since both sets contain exactly one polynomial of degree $n$ for each $n \geq 0$.

Proposition 5.1. For $n>0$ we have

$$
x^{n}=\sum_{k=0}^{\left\lfloor\frac{n-1}{2}\right\rfloor}\left(\begin{array}{c}
n-1-k \\
k
\end{array}\right) \widetilde{Q}_{n-2 k}(x) .
$$

Proof. We claim that the right hand side is the total weight of all lattice paths from $(0,0)$ to some point whose first coordinate is $n$, using northeast steps $(1,1)$ of weight $x$, southeast steps $(1,-1)$ of weight $-1 / x$, and "long" horizontal steps $(2,0)$ of weight 1 , such that the entire path remains strictly above the horizontal axis. Indeed, the first step of each such lattice path is a northeast step. After this step, we select the number $k \in[0,\lfloor(n-1) / 2\rfloor]$ of horizontal steps. By removing the horizontal steps from the list of steps, we obtain a lattice path with altogether $n-2 k$ nonhorizontal steps that remain strictly above the horizontal axis. The total weight of all such lattice paths is $\widetilde{Q}_{n-2 k}(x)$. For each such lattice path, there are $\left(\left(\begin{array}{c}n-2 k \\ k\end{array}\right)\right)=\left(\begin{array}{c}n-k-1 \\ k\end{array}\right)$ ways to reinsert the $k$ horizontal steps after the first, second, $\ldots$, or $(n-2 k)$-th nonhorizontal step.

We only need to show that the total weight of the lattice paths described above is $x^{n}$. The weight of the lattice path consisting of $n$ northeast steps is $x^{n}$. We will show that all other lattice paths may be arranged into pairs whose weights cancel. For that purpose, consider any lattice path $\Gamma$ that contains at least one southeast step or at least one horizontal step. If $\Gamma$ contains a southeast step before any horizontal step, then this step must be immediately preceded by a northeast step. Let $i=i(\Gamma)$ be the least integer such that the step starting at the first coordinate $i$ is either a horizontal step, or it is a northeast step immediately followed by a southeast step. Let $\jmath(\Gamma)$ be the lattice path obtained from $\Gamma$ as follows. If the step starting at the first coordinate $i(\Gamma)$ is a horizontal step, replace it with a northeast step followed by a southeast step. If it is a northeast step followed by a southeast step, replace these two steps with a single horizontal step. The bijection $\Gamma \mapsto \jmath(\Gamma)$ is a fixed point free involution on the set of lattice paths considered and the weight of $\Gamma$ is the negative of the weight of $\jmath(\Gamma)$.

We may rewrite Proposition 5.1 as

$$
x^{2 n}=\sum_{k=1}^{n}\left(\begin{array}{c}
n-1+k \\
n-k
\end{array}\right) \widetilde{Q}_{2 k}(x)
$$

for even powers of $x$ and as

$$
x^{2 n+1}=\sum_{k=0}^{n}\left(\begin{array}{l}
n+k \\
n-k
\end{array}\right) \widetilde{Q}_{2 k+1}(x)
$$


for odd powers of $x$. The coefficients appearing in equations (19) and (20) respectively are exactly the coefficients of the Morgan-Voyce polynomials $B_{n}(x)$ and $b_{n}(x)$ respectively. They first appeared in the study of electrical networks [17]; some of the other early references include Swamy's work [24] and 25]. Another connection between the toric $g$-polynomials of the cubes and the Morgan-Voyce polynomials was noted by Hetyei [11.

Corollary 5.2. The linear transformation $\mathbb{Q}[x] \rightarrow \mathbb{Q}[x]$ given by $x^{n} \mapsto \widetilde{Q}_{n}(x)$ takes $B_{n}\left(x^{2}\right)$ into $x^{2 n}$ and $x b_{n}\left(x^{2}\right)$ into $x^{2 n+1}$.

Comparing Proposition 4.4 with (18) yields the following consequence.

Corollary 5.3. The operators $\mathcal{C}$ and $\mathcal{D}$ are equivalently given by

$$
\mathcal{C}\left(\widetilde{Q}_{n}(x)\right):=\widetilde{Q}_{n+1}(x) \quad \text { and } \quad \mathcal{D}\left(\widetilde{Q}_{n}(x)\right)= \begin{cases}0 & \text { for odd } n, \\ (-1)^{n / 2} C_{n / 2} & \text { for even } n .\end{cases}
$$

We now turn to the polynomials $t_{n}(x):=\mathrm{t}\left(\widehat{B}_{n+1}, x\right)$. Stanley's result [20, Proposition 2.1] may be rewritten as

$$
t_{n}(x)=\sum_{k=0}^{\left\lfloor\frac{n}{2}\right\rfloor} x^{n-2 k} \quad \text { for } n \geq 0 .
$$

Inverting the summation given in (21) yields

$$
x^{n}= \begin{cases}t_{n}(x)-t_{n-2}(x) & \text { if } n \geq 2, \\ t_{n}(x) & \text { if } n \in\{0,1\} .\end{cases}
$$

As an immediate consequence of Definition 4.6 and (22) we obtain

$$
\mathcal{C}\left(t_{n}(x)\right)=t_{n+1}(x)-t_{n-1}(x) \quad \text { and } \quad \mathcal{D}\left(t_{n}(x)\right)=\delta_{n, 0} \quad \text { for } n \geq 0 .
$$

Here we set $t_{-1}(x):=0$ and $\delta_{n, 0}$ is the Kronecker delta function. Finally, the most remarkable property of the basis $\left\{t_{n}(x)\right\}_{n \geq 0}$ is its role in the following result connecting the polynomials $g(P, x)$ and $\mathrm{t}(P, x)$.

Proposition 5.4. Let $[\widehat{0}, \widehat{1}]$ be any Eulerian poset of rank $n+1$. Then

$$
\mathrm{t}([\widehat{0}, \widehat{1}), x)=\sum_{k=0}^{\left\lfloor\frac{n}{2}\right\rfloor} c_{k} t_{n-2 k}(x)
$$

holds for some integers $c_{0}, c_{1}, \ldots, c_{\lfloor n / 2\rfloor}$ if and only if the same integers satisfy

$$
g(\widehat{0}, \widehat{1}), x)=\sum_{k=0}^{\left\lfloor\frac{n}{2}\right\rfloor} c_{k} x^{k} .
$$

Proof. Assuming (24), equation (25) is an immediate consequence of Lemma 3.3 and the obvious identity

$$
U_{\geq 1}\left(\left(x-\frac{1}{x}\right) t_{m}(x)\right)=x^{m+1} .
$$


Conversely, assume (25). Then, it is easy to derive from (77) that

$$
U_{\leq\lfloor n / 2\rfloor} f([\widehat{0}, \widehat{1}), x)=\sum_{k=0}^{\left\lfloor\frac{n}{2}\right\rfloor}\left(\sum_{i=0}^{k} c_{i}\right) x^{k},
$$

from which (24) follows by the last part of (9).

\section{The toric $h$-VeCtor associated to An Eulerian duAl Simplicial Poset}

In this section we use our formulas to compute the toric $h$-vector entries associated to an Eulerian dual simplicial poset in terms of its $f$-vector. This question was mentioned by Stanley [20, citing an observation of Kalai.

Given any graded poset $P$ of rank $n+1$, let us introduce $f_{i}$ for the number of elements of rank $i+1$ in $P$ and call the resulting vector $\left(f_{-1}, f_{0}, \ldots, f_{n}\right)$ the $f$ vector of $P$. If $P$ consists of the faces of a polytope or simplicial complex, ordered by inclusion, then $f_{i}$ is the number of faces of dimension $i$. A graded poset $P$ is simplicial if for all $t \in P \backslash\{\widehat{1}\}$, the interval $[\widehat{0}, t]$ is a Boolean algebra. For the toric $h$-polynomial associated to a graded Eulerian simplicial poset $[\widehat{0}, \widehat{1}]$ of $\operatorname{rank} n+1$, Stanley has the formula [20, Corollary 2.2]

$$
f([\widehat{0}, \widehat{1}), x)=\sum_{i=0}^{n} f_{i-1}(x-1)^{n-i} .
$$

In particular, if $[\widehat{0}, \widehat{1})$ is also the face poset of a simplicial complex, the toric $h$-vector of $P$ coincides with the $h$-vector of the simplicial complex.

A graded poset $P$ is dual simplicial if its dual $P^{*}$, obtained by reversing the partial order, is a simplicial poset. Equivalently, for all $t \in P \backslash\{\widehat{0}\}$, the interval $[t, \widehat{1}]$ is a Boolean algebra. It was first observed by Kalai that the coefficients of the toric $h$-polynomial of a dual simplicial graded Eulerian poset $P$ depend only on the entries $f_{i}$ in the $f$-vector $P$. This linear combination is not unique, and a simple explicit formula was not known before. Given a dual simplicial Eulerian poset $P=[\widehat{0}, \widehat{1}]$, we will express the toric $h$-polynomial coefficients of $[\widehat{0}, \widehat{1})$ in terms of the toric $h$-vector $\left(h_{0}, \ldots, h_{n}\right)$ of the simplicial poset $(\widehat{0}, \widehat{1}]^{*}$. Since $f_{i}(P)=f_{n-1-i}\left(P^{*}\right)$, by (27) the entries of this $h$-vector are given by

$$
h_{k}=\sum_{i=k}^{n}(-1)^{i-k}\left(\begin{array}{l}
i \\
k
\end{array}\right) f_{i}(P) .
$$

Thus, our formulas will show how the toric $h$-polynomial of a dual simplicial poset $P$ depends on the numbers $f_{i}(P)$. It is worth noting that in the special case when $P$ is the face lattice of a simple polytope, the invariants $h_{k}$ given above are the entries of the $h$-vector of the simple polytope. We will apply the following result of Stanley [19, Theorem 3.1] to $P^{*}$.

Theorem 6.1 (Stanley). For each $n$, there exist cd-polynomials $\check{\Phi}_{i}^{n}$ (for $i=$ $0, \ldots, n-1)$ such that the cd-index $\Phi_{P}(c, d)$ of any graded Eulerian simplicial poset $P$ of rank $n+1$ may be written as

$$
\Phi_{P}(c, d)=\sum_{i=0}^{n-1} h_{i} \cdot \check{\Phi}_{i}^{n},
$$

where $\left(h_{0}, \ldots, h_{n}\right)$ is the (toric) h-vector of $P$. 
The following description of the $c d$-polynomials $\check{\Phi}_{i}^{n}$ was conjectured by Stanley [19, Conjecture 3.1] and shown by Hetyei [10, Theorem 2]:

Theorem 6.2. For each $n>0$ and each $0 \leq i \leq n-1$, the polynomial $\check{\Phi}_{i}^{n}$ is the sum of the cd-variation monomials of all augmented André permutations $\pi$ of the set $[1, n+1]$, ordered by the natural order, satisfying $\pi(n)=n-i$.

For our purposes, augmented André permutations are most conveniently defined as follows; see [10, Corollary 1].

Definition 6.3. Let $X$ be any linearly ordered set with $n$ elements. A permutation $\pi$ of $X$ is a word $\pi(1) \cdots \pi(n)$ in which each letter of $X$ occurs exactly once. An augmented André permutation of $X$ is defined recursively as follows:

(i) For $n=0$ the empty word is an augmented André permutation.

(ii) For $n=1$ the only permutation of $X$ is an augmented André permutation.

(iii) For $n>1$, a permutation $\pi$ of $X$ is an augmented André permutation if and only if for $m:=\pi^{-1}(\min X)$ the permutations $\pi(1) \cdots \pi(m-1)$ and $\pi(m+1) \cdots \pi(n)$ are augmented André permutations and the letter $\max (X)$ belongs to $\pi(m+1) \cdots \pi(n)$. Here $\min (X)$, respectively $\max (X)$, is the least, respectively largest, letter of $X$.

For further equivalent definitions and a detailed bibliography on augmented André permutations we refer the reader to [10]. Given a permutation $\pi=\pi(1) \cdots \pi(n)$, the position $i \in[1, n-1]$ is a descent if $\pi(i)>\pi(i+1)$; otherwise it is an ascent. It is stated in one of the equivalent definitions of augmented André permutations [10, Definition 3] that every descent must be followed by an ascent. The cd-variation monomial of an augmented André permutation $\pi(1) \cdots \pi(n)$ is obtained by placing a letter $d$ (of degree 2) to cover the positions $\{i, i+1\}$ for each descent $i$, and covering all remaining positions in $[1, n-1]$ with a letter $c$ (of degree 1 ). As an immediate consequence of Definition 6.3 we obtain that each augmented André permutation must end with the largest letter. Furthermore, we have the following recurrence.

Lemma 6.4. For $n \geq 2$, the noncommutative polynomials $\check{\Phi}_{i}^{n}$ satisfy

$$
\check{\Phi}_{i}^{n}= \begin{cases}\Phi_{\widehat{B}_{n-1}}(c, d) d & \text { if } i=n-1, \\
c \check{\Phi}_{i}^{n-1}+\sum_{m=2}^{n-1} \sum_{j=0}^{\min (i, m-1)}\left(\begin{array}{l}
i \\
j
\end{array}\right)\left(\begin{array}{c}
n-i-2 \\
m-1-j
\end{array}\right) \Phi_{\widehat{B}_{m-1}}(c, d) d \check{\Phi}_{i-j}^{n-m} & \text { if } i<n-1 .\end{cases}
$$

Here $\Phi_{\widehat{B}_{k}}(c, d)$ denotes the $c d$-index of the Boolean algebra of rank $k$.

Proof. An essentially equivalent statement may be found in [10, Proposition 7]. Thus we only outline the proof of this lemma. An augmented André permutation $\pi(1) \cdots \pi(n+1)$ of $[1, n+1]$ must satisfy $\pi(n+1)=n+1$ and in $\check{\Phi}_{i}^{n}$ we consider only André permutations also satisfying $\pi(n)=n-i$. Introducing $m:=\pi^{-1}(1)$ we have $m=n$ if $i=n-1$ and $m \in[1, n-1]$ otherwise. The case $i=n-1$ follows immediately from the definitions. In the case $i<n-1$, term $c \check{\Phi}_{i}^{n}$ is contributed by the augmented André permutations $\pi(1) \cdots \pi(n+1)$ satisfying $m=1$. The variable $j$ in the subsequent double sum stands for the size of the set $\{n-i+1, \ldots, n\} \cap$ $\{\pi(1), \ldots, \pi(m-1)\}$. Besides these $j$ elements, the set $\{\pi(1), \ldots, \pi(m-1)\}$ contains $m-1-j$ further elements of the set $\{2, \ldots, n-i-1\}$. 
As a consequence of Theorems 4.7 and Theorem 6.1 we obtain the following statement.

Corollary 6.5. The short toric polynomial $\mathrm{t}([\widehat{0}, \widehat{1}), x)$ associated to a graded dual simplicial Eulerian poset $P=[\widehat{0}, \widehat{1}]$ of rank $n+1$ may be written as

$$
\mathrm{t}([\widehat{0}, \widehat{1}), x)=\sum_{i=0}^{n-1} h_{i} \check{\Phi}_{i}^{n}(\mathcal{C}, \mathcal{D})(1) .
$$

Here $\left(h_{0}, \cdots, h_{n}\right)$ is the toric h-vector of $(P \backslash\{\widehat{0}\})^{*}$ and $\check{\Phi}_{i}^{n}(\mathcal{C}, \mathcal{D}): \mathbb{Q}[x] \rightarrow \mathbb{Q}[x]$ is the linear operator obtained by replacing each $c$ with $\mathcal{C}$ and each $d$ with $\mathcal{D}$ in the cd-polynomial $\check{\Phi}_{i}^{n}$.

Note that Theorem 4.7 involves reversing the order of the letters when replacing $c$ with $\mathcal{C}$ and $d$ with $\mathcal{D}$. This change, however, is offset by the fact that the $c d-$ index of $P$ is obtained from the $c d$-index of the simplicial poset $P^{*}$ by reversing the order of the letters in each $c d$-monomial. We want to combine Lemma 6.4 with Corollary 6.5 to compute the short toric polynomial of a dual simplicial poset. This will lead to a recurrence expressing the short toric polynomial contributions in terms of the short toric polynomials of the Boolean algebras. As a special case of Corollary 6.5 we obtain the equation

$$
t_{n}(x)=\Phi_{\widehat{B}_{n+1}}(\mathcal{C}, \mathcal{D})(1) .
$$

Here $\Phi_{\widehat{B}_{n+1}}(\mathcal{C}, \mathcal{D})(1)$ is the operator obtained by replacing each $c$ with $\mathcal{C}$ and each $d$ with $\mathcal{D}$ in the $c d$-index $\Phi_{\widehat{B}_{n+1}}(c, d)$ of the Boolean algebra of rank $n+1$.

Theorem 6.6. The short toric polynomial $\mathrm{t}([\widehat{0}, \widehat{1}), x)$ associated to a graded dual simplicial Eulerian poset $P=[\widehat{0}, \widehat{1}]$ of rank $n+1$ may be written as

$$
\begin{aligned}
\mathrm{t}([\widehat{0}, \widehat{1}), x) & =h_{0}\left(t_{n}(x)-(n-1) t_{n-2}(x)\right) \\
& +\sum_{i=1}^{n-1} h_{i} \sum_{k=1}^{\left\lfloor\frac{n}{2}\right\rfloor}\left(\left(\begin{array}{c}
n-i \\
k
\end{array}\right)\left(\begin{array}{c}
i-1 \\
k-1
\end{array}\right)-\left(\begin{array}{c}
n-i-1 \\
k
\end{array}\right)\left(\begin{array}{c}
i \\
k-1
\end{array}\right)\right) t_{n-2 k}(x) .
\end{aligned}
$$

Here $\left(h_{0}, \cdots, h_{n}\right)$ is the toric h-vector of $(P \backslash\{\widehat{0}\})^{*}$.

Proof. By Corollary 6.5 we only need to show that, for each $i \in\{0, \ldots, n-1\}$, the polynomial $t_{n, i}(x):=\check{\Phi}_{i}^{n}(\mathcal{C}, \mathcal{D})(1)$ satisfies

$$
t_{n, i}(x)= \begin{cases}t_{n}(x)-(n-1) t_{n-2}(x) & \text { if } i=0, \\
\sum_{k=1}^{\left\lfloor\frac{n}{2}\right\rfloor}\left(\left(\begin{array}{c}
n-i \\
k
\end{array}\right)\left(\begin{array}{c}
i-1 \\
k-1
\end{array}\right)-\left(\begin{array}{c}
n-i-1 \\
k
\end{array}\right)\left(\begin{array}{c}
i \\
k-1
\end{array}\right)\right) t_{n-2 k}(x) & \text { if } 1 \leq i \leq n-1 .\end{cases}
$$

As before, in the formula above we use the convention $t_{-1}(x)=0$. For small values of $n$ and $i$ the polynomials $t_{n, i}(x)$ are easy to compute using (23) and the list of all polynomials $\check{\Phi}_{i}^{n}$ satisfying $n \leq 5$, published by Stanley [19]. The polynomials $t_{n, i}(x)$, up to $n=5$, are listed in Table 1.

We will prove (29) in several steps, by showing partial statements using induction. In all such arguments our induction step will depend on Lemma 6.4, combined with (28). For $i=n-1$, the combination of these two statements yields

$$
t_{n, n-1}(x)=t_{n-2}(x),
$$


TABLE 1 . The polynomials $t_{n, i}(x)$ for $n \leq 5$.

\begin{tabular}{l||l|l|l|l|l} 
& $i=0$ & $i=1$ & $i=2$ & $i=3$ & $i=4$ \\
\hline \hline$n=1$ & $t_{1}(x)$ & & & \\
$n=2$ & $t_{2}(x)-t_{0}(x)$ & $t_{0}(x)$ & & & \\
$n=3$ & $t_{3}(x)-2 t_{1}(x)$ & $t_{1}(x)$ & $t_{1}(x)$ & & \\
$n=4$ & $t_{4}(x)-3 t_{2}(x)$ & $t_{2}(x)-t_{0}(x)$ & $t_{2}(x)+t_{0}(x)$ & $t_{2}(x)$ & \\
$n=5$ & $t_{5}(x)-4 t_{3}(x)$ & $t_{3}(x)-3 t_{1}(x)$ & $t_{3}(x)+t_{1}(x)$ & $t_{3}(x)+2 t_{1}(x)$ & $t_{3}(x)$
\end{tabular}

whereas for $i<n-1$ we obtain

$t_{n, i}(x)=\mathcal{C}\left(t_{n-1, i}(x)\right)+\sum_{m=2}^{n-1} \sum_{j=0}^{\min (i, m-1)}\left(\begin{array}{l}i \\ j\end{array}\right)\left(\begin{array}{c}n-i-2 \\ m-1-j\end{array}\right) \mathcal{D}\left(t_{n-m, i-j}(x)\right) \cdot t_{m-2}(x)$.

Using these formulas we first prove the case $i=0$ in equation (29). The induction basis is $t_{1,0}(x)=t_{1}(x)$; see Table1, For $n>1$, the recurrence (31) may be rewritten as

$$
t_{n, 0}(x)=\mathcal{C}\left(t_{n-1,0}(x)\right)+\sum_{m=2}^{n-1}\left(\begin{array}{c}
n-2 \\
m-1
\end{array}\right) \mathcal{D}\left(t_{n-m, 0}(x)\right) \cdot t_{m-2}(x) .
$$

This recurrence, combined with the induction hypothesis yields

$$
\begin{aligned}
t_{n, 0}(x)= & \mathcal{C}\left(t_{n-1}(x)-(n-2) t_{n-3}(x)\right) \\
& +\sum_{m=2}^{n-1}\left(\begin{array}{c}
n-2 \\
m-1
\end{array}\right) \mathcal{D}\left(t_{n-m}(x)-(n-m-1) \cdot t_{n-m-2}(x)\right) \cdot t_{m-2}(x) .
\end{aligned}
$$

Using (23) this may be simplified to

$$
\begin{aligned}
t_{n, 0}(x) & =t_{n}(x)-(n-1) t_{n-2}(x)+(n-2) t_{n-4}(x)+\left(\begin{array}{c}
n-2 \\
1
\end{array}\right) t_{n-4}(x) \\
& =t_{n}(x)-(n-1) t_{n-2}(x),
\end{aligned}
$$

which is exactly what we wanted to prove.

Next we observe that all polynomials $t_{n, i}(x)$ are of the form

$$
t_{n, i}(x)=\sum_{k=0}^{\left\lfloor\frac{n}{2}\right\rfloor} \tau_{n, i, k} \cdot t_{n-2 k}(x)
$$

for some integers $\tau_{n, i, k} \in \mathbb{Z}$. This may be easily shown by induction on $n$, using the formulas (30) and (31) and the following two observations:

(1) When we apply the operator $\mathcal{C}$ to an integer linear combination of polynomials $t_{l}(x)$ whose indices are all of the same parity, we obtain an integer linear combination of polynomials $t_{l}(x)$, and all indices are of the opposite parity.

(2) To obtain a nonzero contribution, the input $t_{n-m, i-j}(x)$ of the operator $\mathcal{D}$ must contain $t_{0}(x)$; thus by the induction hypothesis $m$ must have the same parity as $n$. The operator $\mathcal{D}$ sends integers into integers.

Next we rewrite the formulas (30) and (31) as recurrences for the coefficients $\tau_{n, i, k}$. To facilitate this task, we extend the definition of $\tau_{n, i, k}$ to $n=0$ and to $i \geq n$ 
(where $n \geq 0$ ) by setting $\tau_{n, i, k}=0$ if $n=0$ or $i \geq n$. For $n \geq 2$ and $i=n-1$, (30) may be rewritten as

$$
\tau_{n, n-1, k}=\delta_{k, 1},
$$

where $\delta_{k, 1}$ is the Kronecker delta function. When rewriting (31) recall that, as observed above, $\mathcal{D}\left(t_{n-m, i-j}(x)\right)=0$ unless $m$ is of the form $n-2 s$ for some $s$ satisfying $1 \leq s \leq\lfloor(n-2) / 2\rfloor$. Thus (31) may be rewritten as

$t_{n, i}(x)=\mathcal{C}\left(t_{n-1, i}(x)\right)+\sum_{s=1}^{\left\lfloor\frac{n-2}{2}\right\rfloor} \sum_{j=0}^{\substack{\min (i, n-2 s-1)}}\left(\begin{array}{l}i \\ j\end{array}\right)\left(\begin{array}{c}n-i-2 \\ n-2 s-1-j\end{array}\right) \mathcal{D}\left(t_{2 s, i-j}(x)\right) \cdot t_{n-2 s-2}(x)$.

Since $n-2 s-2=n-2 k$ is equivalent to $s=k-1$, comparing coefficients of $t_{n-2 k}$ on both sides of the equation above yields

$$
\tau_{n, i, k}=\tau_{n-1, i, k}-\tau_{n-1, i, k-1}+\sum_{j=0}^{\min (i, n-2 k+1)}\left(\begin{array}{l}
i \\
j
\end{array}\right)\left(\begin{array}{c}
n-i-2 \\
n-2 k+1-j
\end{array}\right) \tau_{2 k-2, i-j, k-1}
$$

for $i<n-1$.

$\tau_{2 k-2, i-j, k-1}=0$ when $k-1=2 k-2=0$ or $i-j \geq 2 k-2$. We only need to show the case $i>0$ of (29), which is equivalent to

$$
\tau_{n, i, k}=\left(\begin{array}{c}
n-i \\
k
\end{array}\right)\left(\begin{array}{c}
i-1 \\
k-1
\end{array}\right)-\left(\begin{array}{c}
n-i-1 \\
k
\end{array}\right)\left(\begin{array}{c}
i \\
k-1
\end{array}\right) \text { for } i>0 .
$$

We prove this statement by induction on $n$, using the polynomials listed in Table 1 as our induction basis. Observe that, for $i=n-1$, (32) gives the same $\delta_{k, 1}$ as (34). Thus we only need to show the validity of (34) in the case when $n \geq 2$ and $i<n-1$, assuming the validity of (29) and, in particular, (34) for all smaller values of $n$. Since $k \leq\lfloor n / 2\rfloor$, we have $2 k-2<n$; thus we may use (33) to compute $\tau_{n, i, k}$.

Case $1(k=0)$. In this case (33) may be simplified to $\tau_{n, i, 0}=\tau_{n-1, i, 0}$. Repeated application of this recurrence yields $\tau_{n, i, 0}=\tau_{i+1, i, 0}$, which equals 0 by (32). Formula (34) also gives 0 .

Case $2(k=1)$. In this case (33) may be simplified to $\tau_{n, i, 1}=\tau_{n-1, i, 1}-\tau_{n-1, i, 0}$. By the already shown previous case (and also by our induction hypothesis) we have $\tau_{n-1, i, 0}=0$; thus we obtain the recurrence $\tau_{n, i, 1}=\tau_{n-1, i, 1}$. Repeated application of this recurrence yields $\tau_{n, i, 1}=\tau_{i+1, i, 1}$, which equals 1 by (32). Formula (34) also gives 1.

Case $3(k=2)$. By Table 1 we have $\tau_{2,0,1}=-1$ and $\tau_{2,1,1}=1$. Thus (33) may be simplified to

$\tau_{n, i, 2}=\tau_{n-1, i, 2}-\tau_{n-1, i, 1}+\left(\begin{array}{c}i \\ i-1\end{array}\right)\left(\begin{array}{l}n-i-2 \\ n-i-2\end{array}\right)-\left(\begin{array}{c}i \\ i\end{array}\right)\left(\begin{array}{c}n-i-2 \\ n-3-i\end{array}\right) \quad$ for $i<n-1$.

This recurrence equals the recurrence obtained by substituting $k=2$ into (35) in Case 4 below. The rest of the proof of this case is identical to the proof of Case 4 .

Case $4(k \geq 3)$. Consider the term $\tau_{2 k-2, i-j, k-1}$ in the sum on the right hand side of (33). If $i-j=0$, then, by the already shown first part of (29) we have

$$
t_{2 k-2,0}(x)=t_{2 k-2}(x)-(2 k-3) t_{2 k-4}(x),
$$


implying $\tau_{2 k-2,0, k-1}=0$, since $k-1 \geq 2$. If $i-j>0$, then, by our induction hypothesis we have

$$
\tau_{2 k-2, i-j, k-1}=\left(\begin{array}{c}
2 k-2-(i-j) \\
k-1
\end{array}\right)\left(\begin{array}{c}
i-j-1 \\
k-2
\end{array}\right)-\left(\begin{array}{c}
2 k-3-(i-j) \\
k-1
\end{array}\right)\left(\begin{array}{l}
i-j \\
k-2
\end{array}\right) .
$$

Here we have $\left(\begin{array}{c}2 k-2-(i-j) \\ k-1\end{array}\right)=\left(\begin{array}{c}2 k-3-(i-j) \\ k-1\end{array}\right)=0$ unless $i-j \leq k-1$ and $\left(\begin{array}{c}i-j-1 \\ k-2\end{array}\right)=$ $\left(\begin{array}{c}i-j \\ k-2\end{array}\right)=0$ unless $i-j \geq k-2$. As a consequence, $\tau_{2 k-2, i-j, k-1}=0$ unless $i-j \in$ $\{k-2, k-1\}$. Direct substitution into the above formula shows $\tau_{2 k-2, k-2, k-1}=-1$ and $\tau_{2 k-2, k-1, k-1}=1$. Therefore, the recurrence (33) may be simplified to

$$
\tau_{n, i, k}=\tau_{n-1, i, k}-\tau_{n-1, i, k-1}+\left(\begin{array}{c}
i \\
i-k+1
\end{array}\right)\left(\begin{array}{l}
n-i-2 \\
n-k-i
\end{array}\right)-\left(\begin{array}{c}
i \\
i-k+2
\end{array}\right)\left(\begin{array}{c}
n-i-2 \\
n-k-1-i
\end{array}\right) .
$$

Substituting our induction hypothesis for $\tau_{n-1, i, k-1}$ and using the symmetry of the binomial coefficients yields

$$
\begin{aligned}
\tau_{n, i, k}= & \tau_{n-1, i, k}-\left(\begin{array}{c}
n-i-1 \\
k-1
\end{array}\right)\left(\begin{array}{c}
i-1 \\
k-2
\end{array}\right)+\left(\begin{array}{c}
n-i-2 \\
k-1
\end{array}\right)\left(\begin{array}{c}
i \\
k-2
\end{array}\right) \\
& +\left(\begin{array}{c}
i \\
k-1
\end{array}\right)\left(\begin{array}{c}
n-i-2 \\
k-2
\end{array}\right)-\left(\begin{array}{c}
i \\
k-2
\end{array}\right)\left(\begin{array}{c}
n-i-2 \\
k-1
\end{array}\right) \\
= & \tau_{n-1, i, k}-\left(\begin{array}{c}
n-i-1 \\
k-1
\end{array}\right)\left(\begin{array}{c}
i-1 \\
k-2
\end{array}\right)+\left(\begin{array}{c}
i \\
k-1
\end{array}\right)\left(\begin{array}{c}
n-i-2 \\
k-2
\end{array}\right) .
\end{aligned}
$$

Substituting our induction hypothesis for $\tau_{n-1, i, k}$ yields

$$
\begin{aligned}
\tau_{n, i, k}= & \left(\begin{array}{c}
n-i-1 \\
k
\end{array}\right)\left(\begin{array}{c}
i-1 \\
k-1
\end{array}\right)-\left(\begin{array}{c}
n-i-2 \\
k
\end{array}\right)\left(\begin{array}{c}
i \\
k-1
\end{array}\right)+\left(\begin{array}{c}
n-i-2 \\
k-2
\end{array}\right)\left(\begin{array}{c}
i \\
k-1
\end{array}\right) \\
& -\left(\begin{array}{c}
n-i-1 \\
k-1
\end{array}\right)\left(\begin{array}{c}
i-1 \\
k-2
\end{array}\right) .
\end{aligned}
$$

By Pascal's identity, we may replace $\left(\begin{array}{l}i-1 \\ k-2\end{array}\right)$ with $\left(\begin{array}{c}i \\ k-1\end{array}\right)-\left(\begin{array}{c}i-1 \\ k-1\end{array}\right)$ and $\left(\begin{array}{c}n-i-2 \\ k-2\end{array}\right)$ with $\left(\begin{array}{c}n-i-1 \\ k-1\end{array}\right)-\left(\begin{array}{c}n-i-2 \\ k-1\end{array}\right)$ in the last equation. Thus we obtain

$$
\begin{aligned}
\tau_{n, i, k}= & \left(\begin{array}{c}
n-i-1 \\
k
\end{array}\right)\left(\begin{array}{c}
i-1 \\
k-1
\end{array}\right)-\left(\begin{array}{c}
n-i-2 \\
k
\end{array}\right)\left(\begin{array}{c}
i \\
k-1
\end{array}\right)+\left(\begin{array}{c}
n-i-1 \\
k-1
\end{array}\right)\left(\begin{array}{c}
i \\
k-1
\end{array}\right) \\
& -\left(\begin{array}{c}
n-i-2 \\
k-1
\end{array}\right)\left(\begin{array}{c}
i \\
k-1
\end{array}\right)-\left(\begin{array}{c}
n-i-1 \\
k-1
\end{array}\right)\left(\begin{array}{c}
i \\
k-1
\end{array}\right)+\left(\begin{array}{c}
n-i-1 \\
k-1
\end{array}\right)\left(\begin{array}{c}
i-1 \\
k-1
\end{array}\right) .
\end{aligned}
$$

After collecting terms by factors of $\left(\begin{array}{c}i-1 \\ k-1\end{array}\right)$ and $\left(\begin{array}{c}i \\ k-1\end{array}\right)$, respectively, and using Pascal's identity, we obtain (34).

An important equivalent form of Theorem 6.6] is the following statement.

Proposition 6.7. Let $[\widehat{0}, \widehat{1}]$ be a graded dual simplicial Eulerian poset of rank $n+1$ and let $\left(h_{0}, \cdots, h_{n}\right)$ be the toric $h$-vector of $(\widehat{0}, \widehat{1}]^{*}$. Then we have

$\mathrm{t}([\widehat{0}, \widehat{1}), x)=h_{0} t_{n}(x)+\sum_{i=1}^{\left\lfloor\frac{n}{2}\right\rfloor}\left(h_{i}-h_{i-1}\right) \sum_{k=1}^{\min \{i, n-i\}} \frac{n+1-2 i}{k}\left(\begin{array}{l}n-i \\ k-1\end{array}\right)\left(\begin{array}{l}i-1 \\ k-1\end{array}\right) t_{n-2 k}(x)$. 
Proof. As an immediate consequence of Theorem 6.6, we have $\left[t_{n}(x)\right] \mathrm{t}([\widehat{0}, \widehat{1}), x)=$ $h_{0}$. We only need to prove that the coefficient of $t_{n-2 k}(x)$ in $\mathrm{t}([\widehat{0}, \widehat{1}), x)$ is correctly stated for $k \geq 1$. In terms of the coefficients $\tau_{n, i, k}$, introduced in the proof of Theorem 6.6. we have

$$
\left[t_{n-2 k}(x)\right] \mathrm{t}([\widehat{0}, \widehat{1}), x)=\sum_{i=0}^{n-1} h_{i} \cdot \tau_{n, i, k} .
$$

Using the fact that $\left(h_{0}, \ldots, h_{n}\right)$ is the toric $h$-vector of the dual of $[\widehat{0}, \widehat{1}]$ and thus satisfies $h_{i}=h_{n-i}$ for $i=1,2, \ldots, n$, we may rewrite the above equation as

$$
\begin{aligned}
{\left[t_{n-2 k}(x)\right] \mathrm{t}([\widehat{0}, \widehat{1}), x)=} & h_{0} \cdot \tau_{n, 0, k}+\sum_{i=1}^{\lfloor n / 2\rfloor} h_{i} \cdot\left(\tau_{n, i, k}+\tau_{n, n-i, k}\right) \\
& -\delta_{\lfloor n / 2\rfloor,\lceil n / 2\rceil} \cdot h_{\lfloor n / 2\rfloor} \cdot \tau_{n,\lfloor n / 2\rfloor, k} .
\end{aligned}
$$

Here $\delta_{\lfloor n / 2\rfloor,\lceil n / 2\rceil}$ is the Kronecker delta, and adding the last term represents subtracting $h_{n / 2} \cdot \tau_{n, n / 2, k}$ exactly when $n$ is even. Rewriting the right hand side as a linear combination of $h_{0}, h_{1}-h_{0}, \ldots, h_{\lfloor n / 2\rfloor}-h_{\lfloor n / 2\rfloor-1}$ yields

$$
\left[t_{n-2 k}(x)\right] \mathrm{t}([\widehat{0}, \widehat{1}), x)=h_{0} \cdot\left(\tau_{n, 0, k}+\sum_{j=1}^{n-1} \tau_{n, j, k}\right)+\sum_{i=1}^{\lfloor n / 2\rfloor}\left(h_{i}-h_{i-1}\right) \cdot \sum_{j=i}^{n-i} \tau_{n, j, k} .
$$

The statement now follows from (29) and from the fact that

$$
\begin{aligned}
\sum_{j=i}^{n-i} \tau_{n, j, k} & =\sum_{j=i}^{n-i}\left(\left(\begin{array}{c}
n-j \\
k
\end{array}\right)\left(\begin{array}{l}
j-1 \\
k-1
\end{array}\right)-\left(\begin{array}{c}
n-j-1 \\
k
\end{array}\right)\left(\begin{array}{c}
j \\
k-1
\end{array}\right)\right) \\
& =\sum_{j=i-1}^{n-i-1}\left(\begin{array}{c}
n-j-1 \\
k
\end{array}\right)\left(\begin{array}{c}
j \\
k-1
\end{array}\right)-\sum_{j=i}^{n-i}\left(\begin{array}{c}
n-j-1 \\
k
\end{array}\right)\left(\begin{array}{c}
j \\
k-1
\end{array}\right) \\
& =\left(\begin{array}{c}
n-i \\
k
\end{array}\right)\left(\begin{array}{c}
i-1 \\
k-1
\end{array}\right)-\left(\begin{array}{c}
i-1 \\
k
\end{array}\right)\left(\begin{array}{l}
n-i \\
k-1
\end{array}\right)=\frac{n+1-2 i}{k}\left(\begin{array}{l}
n-i \\
k-1
\end{array}\right)\left(\begin{array}{c}
i-1 \\
k-1
\end{array}\right)
\end{aligned}
$$

holds for $i=1,2, \ldots,\lfloor n / 2\rfloor$. Note that, applying the last equation to $i=1$ implies

$$
\begin{aligned}
\tau_{n, 0, k}+\sum_{j=1}^{n-1} \tau_{n, j, k} & =\tau_{n, 0, k}+\frac{n-1}{k}\left(\begin{array}{l}
n-1 \\
k-1
\end{array}\right)\left(\begin{array}{c}
0 \\
k-1
\end{array}\right) \\
& = \begin{cases}-(n-1)+(n-1) & \text { for } k=1, \\
0+0 & \text { for } k \geq 2 .\end{cases}
\end{aligned}
$$

In either case, $h_{0}$ contributes zero to $t_{n-2 k}(x)$ for $k \geq 1$.

Using Proposition [5.4. Theorem 6.6 and Proposition 6.7, respectively, may be rewritten as the formulas stated in the next two corollaries.

Corollary 6.8. Let $[\widehat{0}, \widehat{1}]$ be a graded dual simplicial Eulerian poset of rank $n+1$ and let $\left(h_{0}, \cdots, h_{n}\right)$ be the toric $h$-vector of $(\widehat{0}, \widehat{1}]^{*}$. Then

$$
\begin{aligned}
g([\widehat{0}, \widehat{1}), x) & =h_{0}(1-(n-1) x) \\
& +\sum_{i=1}^{n-1} h_{i} \sum_{k=1}^{\left\lfloor\frac{n}{2}\right\rfloor}\left(\left(\begin{array}{c}
n-i \\
k
\end{array}\right)\left(\begin{array}{c}
i-1 \\
k-1
\end{array}\right)-\left(\begin{array}{c}
n-i-1 \\
k
\end{array}\right)\left(\begin{array}{c}
i \\
k-1
\end{array}\right)\right) x^{k} .
\end{aligned}
$$


Corollary 6.9. Let $[\widehat{0}, \widehat{1}]$ be a graded dual simplicial Eulerian poset of rank $n+1$ and let $\left(h_{0}, \cdots, h_{n}\right)$ be the toric $h$-vector of $(\widehat{0}, \widehat{1}]^{*}$. Then

$$
g(\widehat{0}, \widehat{1}), x)=h_{0}+\sum_{i=1}^{\left\lfloor\frac{n}{2}\right\rfloor}\left(h_{i}-h_{i-1}\right) \sum_{k=1}^{\min \{i, n-i\}} \frac{n+1-2 i}{k}\left(\begin{array}{c}
n-i \\
k-1
\end{array}\right)\left(\begin{array}{c}
i-1 \\
k-1
\end{array}\right) x^{k}
$$

The most important consequence of Corollary 6.9 is the following.

Corollary 6.10. Let $[\widehat{0}, \widehat{1}]$ be a graded dual simplicial Eulerian poset of rank $n+1$. If the toric $h$-vector $\left(h_{0}, \ldots, h_{n}\right)$ of $(\widehat{0}, \widehat{1}]^{*}$ satisfies $h_{0} \leq h_{1} \leq \cdots \leq h_{\lfloor n / 2\rfloor}$, then $f([\widehat{0}, \widehat{1}], x)$ has nonnegative coefficients.

Indeed, by Corollary 6.9 above, $g([\widehat{0}, \widehat{1}), x)$ has nonnegative coefficients and the statement follows from (11).

Example 6.11. Let $[\widehat{0}, \widehat{1}]$ be the face lattice of an $n$-dimensional simple polytope $\mathcal{P}$. This is an Eulerian dual simplicial poset of rank $n+1$; its dual is the face lattice of a simplicial polytope. The coefficients of $f([\widehat{0}, \widehat{1}], x)$ form the toric $h$-vector of $\mathcal{P}$. By Corollary 6.10, the fact that the toric $h$-vector of $\mathcal{P}$ has nonnegative entries is a consequence of the Generalized Lower Bound Theorem [21] for simplicial polytopes.

Remark 6.12. As pointed out by Christian Krattenthaler [15], the coefficient

$$
\frac{n+1-2 i}{k}\left(\begin{array}{c}
n-i \\
k-1
\end{array}\right)\left(\begin{array}{c}
i-1 \\
k-1
\end{array}\right)=\left(\begin{array}{c}
n+1-i \\
k
\end{array}\right)\left(\begin{array}{l}
i-1 \\
k-1
\end{array}\right)-\left(\begin{array}{l}
n-i \\
k-1
\end{array}\right)\left(\begin{array}{l}
i \\
k
\end{array}\right)
$$

of $x^{k}$ in the contribution of $h_{i}-h_{i-1}$ to the right hand side of Corollary 6.9 is the number of lattice paths from $(0,0)$ to $(n+1-i, i)$ using $N$ steps $(1,0)$ and $E$ steps $(0,1)$ with exactly $k$ NE turns, such that the lattice path does not cross the line $y=x$ and has its last NE turn on the line $y=i$. In particular, in the case when $n=2 i$, the coefficients $N(i, k)=\left(\begin{array}{c}i-1 \\ k-1\end{array}\right)\left(\begin{array}{c}i \\ k-1\end{array}\right) / k$, contributed by $h_{\lfloor n / 2\rfloor}-h_{\lfloor n / 2\rfloor-1}$, are the Narayana numbers. The above description may be shown with a slight modification of the proof of [14, Theorem 3.4.1]. A direct application of [14, Theorem 3.4.1] yields that, for $i \geq(n-1) / 2$, the coefficients

$$
\left(\begin{array}{c}
n-i \\
k
\end{array}\right)\left(\begin{array}{c}
i-1 \\
k-1
\end{array}\right)-\left(\begin{array}{c}
n-i-1 \\
k
\end{array}\right)\left(\begin{array}{c}
i \\
k-1
\end{array}\right)=\left(\begin{array}{c}
n-i-1 \\
k-1
\end{array}\right)\left(\begin{array}{c}
i \\
k-1
\end{array}\right)-\left(\begin{array}{c}
n-i \\
k
\end{array}\right)\left(\begin{array}{c}
i-1 \\
k-2
\end{array}\right)
$$

contributed by $h_{i}$ in Corollary 6.8, count all lattice paths from $(0,0)$ to $(i, n-i-1)$ using $N$ steps and $E$ steps with exactly $k-1$ NE turns, such that the lattice path does not cross the line $y=x$. In particular, the coefficients of the contributions of $h_{\lfloor n / 2\rfloor}$ and $h_{\lceil n / 2\rceil}$ are again the Narayana numbers. For a detailed bibliography of the Narayana numbers we refer to sequence A001263 in the On-Line Encyclopedia of Integer Sequences [18. For $i<(n-1) / 2$, the contributions of $h_{i}$ in Corollary 6.8 also contain negative coefficients; it seems hard to see a pattern of signs.

Motivated by the well-known example of the cube (see Example 6.14 below), we rewrite Corollary 6.8 in the basis $\left\{(x-1)^{k}\right\}_{k \geq 0}$. 
Proposition 6.13. Let $[\widehat{0}, \widehat{1}]$ be a graded dual simplicial Eulerian poset of rank $n+1$, and let $\left(h_{0}, \cdots, h_{n}\right)$ be the toric $h$-vector of $(\widehat{0}, \widehat{1}]^{*}$. Then we have

$$
\begin{aligned}
g([\widehat{0}, \widehat{1}), x) & =h_{0}(-n+2-(n-1)(x-1)) \\
& +\sum_{i=1}^{n-1} h_{i} \sum_{k=0}^{\left\lfloor\frac{n}{2}\right\rfloor}\left(\left(\begin{array}{c}
n-i \\
k
\end{array}\right)\left(\begin{array}{c}
n-k-1 \\
i-k
\end{array}\right)-\left(\begin{array}{c}
n-i-1 \\
k
\end{array}\right)\left(\begin{array}{c}
n-k-1 \\
i+1-k
\end{array}\right)\right)(x-1)^{k} .
\end{aligned}
$$

Proof. The contribution of $h_{0}$ is the same in the above formula and in Corollary 6.8, We only need to verify that each $h_{i}$ has the same contribution when $i>0$. By the binomial theorem and the symmetry of the binomial coefficients, when we expand the contribution of $h_{i}$ given in Corollary 6.8 in the basis $\left\{(x-1)^{k}\right\}_{k \geq 0}$, the coefficient of $(x-1)^{k}$ is

$$
\sigma_{n, i, k}:=\sum_{j=k}^{\left\lfloor\frac{n}{2}\right\rfloor}\left(\begin{array}{l}
j \\
k
\end{array}\right)\left(\left(\begin{array}{c}
n-i \\
j
\end{array}\right)\left(\begin{array}{l}
i-1 \\
i-j
\end{array}\right)-\left(\begin{array}{c}
n-i-1 \\
j
\end{array}\right)\left(\begin{array}{c}
i \\
i+1-j
\end{array}\right)\right) .
$$

Using the identity $\left(\begin{array}{c}n \\ m\end{array}\right)\left(\begin{array}{c}m \\ k\end{array}\right)=\left(\begin{array}{c}n \\ k\end{array}\right)\left(\begin{array}{c}n-k \\ m-k\end{array}\right)$ we obtain

$$
\begin{aligned}
\sigma_{n, i, k}= & \left(\begin{array}{c}
n-i \\
k
\end{array}\right) \sum_{j=k}^{\left\lfloor\frac{n}{2}\right\rfloor}\left(\begin{array}{c}
n-i-k \\
j-k
\end{array}\right)\left(\begin{array}{c}
i-1 \\
i-j
\end{array}\right) \\
& -\left(\begin{array}{c}
n-i-1 \\
k
\end{array}\right) \sum_{j=k}^{\left\lfloor\frac{n}{2}\right\rfloor}\left(\begin{array}{c}
n-i-1-k \\
j-k
\end{array}\right)\left(\begin{array}{c}
i \\
i+1-j
\end{array}\right) .
\end{aligned}
$$

The statement now follows from the Chu-Vandermonde identity. Note that the terms in the last two sums are zero unless $j \leq \min (i+1, n-i-1)$, which is a more stringent condition than $j \leq\lfloor n / 2\rfloor$.

Using Pascal's identity and the symmetry of the binomial coefficients, Proposition 6.13 yields

$$
\begin{aligned}
\sigma_{n, i, k}= & \left(\begin{array}{c}
n-i-1 \\
k
\end{array}\right)\left(\begin{array}{c}
n-k-1 \\
n-i-1
\end{array}\right)+\left(\begin{array}{c}
n-i-1 \\
k-1
\end{array}\right)\left(\begin{array}{c}
n-k-1 \\
n-i-1
\end{array}\right) \\
& -\left(\begin{array}{c}
n-i-2 \\
k
\end{array}\right)\left(\begin{array}{c}
n-k-1 \\
n-i-2
\end{array}\right)-\left(\begin{array}{c}
n-i-2 \\
k-1
\end{array}\right)\left(\begin{array}{c}
n-k-1 \\
n-i-2
\end{array}\right)
\end{aligned}
$$

for $i>0$ and the coefficients $\sigma_{n, i, k}$ defined in (36). This may be rewritten as

$$
\begin{aligned}
\sigma_{n, i, k}= & \left(\begin{array}{c}
n-k-1 \\
k
\end{array}\right) p(n-2 k-1, n-i-k-1) \\
& +\left(\begin{array}{c}
n-k-1 \\
k-1
\end{array}\right) p(n-2 k, n-i-k) \quad \text { for } i>0,
\end{aligned}
$$

where we follow the notation of Bayer and Ehrenborg [3] by setting $p(n, k):=$ $\left(\begin{array}{l}n \\ k\end{array}\right)-\left(\begin{array}{c}n \\ k-1\end{array}\right)$.

Example 6.14. Consider the face lattice $\widehat{L_{n}}$ of an $n$-dimensional cube. This is a graded, dual simplicial Eulerian poset of rank $n+1$, satisfying $h_{i}=\left(\begin{array}{c}n \\ i\end{array}\right)$. Thus (37) 
and the Chu-Vandermonde identity yield

$\sum_{i=1}^{n-1}\left(\begin{array}{c}n \\ i\end{array}\right) \sigma_{n, i, k}=\left(\begin{array}{c}n-k-1 \\ k\end{array}\right) p(2 n-2 k-1, n-k-1)+\left(\begin{array}{c}n-k-1 \\ k-1\end{array}\right) p(2 n-2 k, n-k)$

for $k \geq 2$. Note that the assumption $k \geq 2$ is necessary to make sure that the omitted substitution $i=0$ yields only zero terms in all applications of the Chu-Vandermonde identity. Using the fact that $p(2 n-2 k-1, n-k-1)$ and $p(2 n-2 k, n-k)$ both equal the Catalan number $C_{n-k}$ we obtain

$$
\sum_{i=1}^{n-1}\left(\begin{array}{c}
n \\
i
\end{array}\right) \sigma_{n, i, k}=\left(\begin{array}{c}
n-k \\
k
\end{array}\right) C_{n-k} .
$$

Similarly, for $k=0$ and $k=1$ respectively, we obtain

$$
\sum_{i=1}^{n-1}\left(\begin{array}{c}
n \\
i
\end{array}\right) \sigma_{n, i, 0}=\left(\begin{array}{c}
n \\
0
\end{array}\right) C_{n}+n-2 \quad \text { and } \quad \sum_{i=1}^{n-1}\left(\begin{array}{c}
n \\
i
\end{array}\right) \sigma_{n, i, 1}=\left(\begin{array}{c}
n-1 \\
1
\end{array}\right) C_{n-1}+n-1 .
$$

The additional terms on the right hand sides account for the omitted substitutions $i=0$ on the left hand sides. Considering that $h_{0}=1$ contributes $-n+2-(n-$ 1) $(x-1)$, we find

$$
g\left(L_{n}, x\right)=\sum_{k=0}^{\lfloor n / 2\rfloor}\left(\begin{array}{c}
n-k \\
k
\end{array}\right) C_{n-k}(x-1)^{k} .
$$

Here $L_{n}$ is obtained from $\widehat{L_{n}}$ by removing its maximum element. It was noted in [11, Lemma 3.3] that the above formula is equivalent to Gessel's result [20, Proposition 2.6], stating

$$
g\left(L_{n}, x\right)=\sum_{k=0}^{\lfloor n / 2\rfloor} \frac{1}{n-k+1}\left(\begin{array}{l}
n \\
k
\end{array}\right)\left(\begin{array}{c}
2 n-2 k \\
n
\end{array}\right)(x-1)^{k} .
$$

The first combinatorial interpretation of the right hand side of (39) is due to Shapiro [22, Ex. 3.71g], the proof of which was published by Chan [9, Proposition 2].

\section{APPENDIX}

Here we outline how to derive a formula expressing $\mathrm{t}(P, x)$ in terms of the flag $h$-vector of an arbitrary poset $P$ that has a unique minimum element $\widehat{0}$ and a rank function $\rho: P \rightarrow \mathbb{N}$, satisfying $\rho(\widehat{0})=0$ and $\max \{\rho(p): p \in P\}=n$. There is no need nor place to use the reflection principle this time. The line of thought present here is essentially an adaptation of the work of Bayer and Ehrenborg 3, Section 7.4].

In analogy to the proof of [3. Theorem 7.14] we begin with substituting (1) into (12) and rearranging. This yields

$$
\mathrm{t}(P, x)=\sum_{\lambda \in\{-1,1\}^{n}: n-2 i_{\lambda} \geq 0} x^{n-2 i_{\lambda}}(-1)^{|S(\lambda)|+n-i_{\lambda}} h_{S(\lambda)} .
$$

This equation may be rewritten as

$$
\mathrm{t}(P, x)=\sum_{S \subseteq[1, n]} h_{S} \cdot \mathrm{t}_{h}(S, x),
$$


where $\mathrm{t}_{h}(S, x)$ is the total weight of all $\lambda \in\{-1,1\}^{n}$ such that $\lambda_{1}+\cdots+\lambda_{n} \geq 0$ and $S(\lambda)=S$. Here each $\lambda_{i}=-1$ contributes a factor of $x$, each $\lambda_{i}=1$ contributes a factor of $-1 / x$, and each $i \in S(\lambda)$ contributes an additional factor of -1 to the weight of $\lambda$. Just as in [3, Section 7.4] and in Section 4 above, we may associate to each $\lambda \in\{-1,1\}^{n}$ a lattice path starting at $(0,0)$, such that each $\lambda_{i}$ is replaced with a step $\left(1, \lambda_{i}\right)$. We may then use this observation to express the polynomials $\mathrm{t}_{h}(S, x)$ in the basis $\left\{\widetilde{Q}_{k}(x)\right\}_{k \geq 0}$. For this purpose we need to recall the notion of the unique sparse interval system $\mathcal{I}[S]$ associated to a set of positive integers $S$; see [8]. An antichain of intervals $\mathcal{I}=\left\{\left[i_{1}, j_{1}\right], \ldots,\left[i_{r}, j_{r}\right]\right\}$ satisfying $i_{1}<\cdots<i_{r}$ is sparse if for all $k \in[1, r-1]$ we have $j_{k}+1<i_{k+1}$. For every set $S$ of positive integers there is a unique sparse interval system $\mathcal{I}$ such that $S$ is the union of the intervals of $\mathcal{I}$. We denote this family of intervals by $\mathcal{I}[S]$. We obtain the following short toric generalization of [3, Theorem 3.1].

Proposition A.15. Let $S \subseteq[1, n]$ be a set given by $\mathcal{I}[S]=\left\{\left[i_{1}, j_{1}\right], \cdots,\left[i_{r}, j_{r}\right]\right\}$. Then the polynomial $\mathrm{t}_{h}(S, x)$ appearing in (40) is given by

$$
\mathrm{t}_{h}(S, x)= \begin{cases}(-1)^{r+\frac{n}{2}} \prod_{k=1}^{r}\left(C_{\frac{j_{k}-i_{k}}{2}} C_{\frac{i_{k}-j_{k-1}}{2}}\right) C_{\frac{n-j_{r}}{2}} & \text { if } j_{r}<n ; \\ (-1)^{r+\frac{i_{r}-1}{2}} \prod_{k=1}^{r-1} C_{\frac{j_{k}-i_{k}}{2}} \prod_{k=1}^{r} C_{\frac{i_{k}-j_{k-1}}{2}} \widetilde{Q}_{n-i_{r}+1}(x) & \text { if } j_{r}=n .\end{cases}
$$

Here we set $j_{0}:=0$.

Note that $n$ must be even if $j_{r}<n$ since $\lambda_{1}+\cdots+\lambda_{n}=0$. Similarly, $i_{r}$ must be odd if $j_{r}=n$.

\section{ACKNOWLEDGMENTS}

The author wishes to thank Margaret Bayer, Louis Billera and Richard Stanley for useful advice and encouragement. Christian Krattenthaler's explanation greatly increased the author's understanding of a combinatorial interpretation of most numbers appearing in formulas for the toric polynomials of a dual simplicial poset. Finally, the author's heartfelt thanks go to an anonymous referee for the very careful reading of this manuscript and for many vital corrections.

\section{REFERENCES}

[1] M. Bayer, Face numbers and subdivisions of convex polytopes, in: "Polytopes: abstract, convex and computational (Scarborough, ON, 1993)," pp. 155-171, NATO Adv. Sci. Inst. Ser. C Math. Phys. Sci., 440, Kluwer Acad. Publ., Dordrecht, 1994. MR.1322061 (96a:52012)

[2] M. Bayer and L. Billera, Generalized Dehn-Sommerville relations for polytopes, spheres and Eulerian partially ordered sets, Invent. Math. 79 (1985), 143-157. MR774533 (86f:52010b)

[3] Margaret M. Bayer and Richard Ehrenborg, The toric $h$-vectors of partially ordered sets, Trans. Amer. Math. Soc. 352 (2000), 4515-4531. MR.1779486 (2001k:52015)

[4] M. Bayer and G. Hetyei, Flag vectors of Eulerian partially ordered sets, European J. Combin. 22 (2001), 5-26. MR.1808080 (2002b:06003)

[5] M. Bayer and G. Hetyei, Generalizations of Eulerian partially ordered sets, flag numbers, and the Möbius function, Discrete Math. 256 (2002), 577-593. MR1935777 (2003g:06006)

[6] M. Bayer and Klapper, A new index for polytopes, Discrete Comput. Geom. 6 (1991), 33-47. MR:1073071 (91k:52024)

[7] L. J. Billera and Francesco Brenti, Quasisymmetric functions and Kazhdan-Lusztig polynomials, preprint 2007, to appear in Israel Journal of Mathematics, arXiv:0710.3965v2 [math.CO]. 
[8] L. J. Billera and G. Hetyei, Decompositions of partially ordered sets, Order 17 (2000), 141166. MR 1795999 (2002m:05199)

[9] Clara S. Chan, Plane trees and $H$-vectors of shellable cubical complexes, SIAM J. Discrete Math. 4 (1991), 568-574. MR.1129393 (92k:05134)

[10] G. Hetyei, On the $c d$-variation polynomials of André and Simsun permutations, Discrete Comput. Geom. 16 (1996), 259-275. MR1410161(97j:06004)

[11] G. Hetyei, A second look at the toric $h$-polynomial of a cubical complex, preprint 2010, arXiv:1002.3601v1 [math.CO], to appear in Ann. Combin.

[12] K. Karu, Hard Lefschetz theorem for nonrational polytopes, Invent. Math. 157 (2004), 419-447. MR2076929(2005k:52023)

[13] K. Karu, The $c d$-index of fans and posets, Compos. Math. 142 (2006), 701-718. MR2231198 (2007e:14083)

[14] C. Krattenthaler, The enumeration of lattice paths with respect to their number of turns, in: "Advances in combinatorial methods and applications to probability and statistics," pp. 29-58, Stat. Ind. Technol., Birkhäuser Boston, Boston, MA, 1997. MR1456725 (98f:05006)

[15] C. Krattenthaler, personal communication.

[16] C. Lee, Sweeping the $c d$-Index and the Toric $H$-Vector, preprint 2009, http: //www.ms .uky . edu $/ \sim$ lee/cd.pdf

[17] A. M. Morgan-Voyce, Ladder Network Analysis Using Fibonacci Numbers, IRE Trans. Circuit Th. CT-6, 321-322, Sep. 1959.

[18] N.J.A. Sloane, "On-Line Encyclopedia of Integer Sequences," http://www.research.att. com/ njas/sequences

[19] R. P. Stanley, Flag $f$-vectors and the $c d$-index, Math. Z. 216 (1994), 483-499. MR.1283084 (96b:06006)

[20] R. P. Stanley, Generalized $H$-Vectors, Intersection Cohomology of Toric Varieties and Related Results, in: "Commutative Algebra and Combinatorics" (M. Nagata and H. Matsumura, eds.), Advanced Studies in Pure Math. 11, Kinokuniya, Tokyo and North-Holland, Amsterdam, New York, Oxford, 1987, 187-213. MR951205(89f:52016)

[21] R. P. Stanley, The number of faces of simplicial polytopes and spheres, in: "Discrete Geometry and Convexity" (J. E. Goodman, et al., eds.), Ann. New York Acad. Sci., vol. 440 (1985), pp. 212-223. MR809209 (87a:52007)

[22] R. P. Stanley, "Enumerative Combinatorics, Volume I," Cambridge University Press, Cambridge, 1997. MR.1442260(98a:05001)

[23] R.P. Stanley, "Enumerative Combinatorics, Volume II," Cambridge University Press, Cambridge, 1999. MR1676282(2000k:05026)

[24] M. N. S. Swamy, Properties of the Polynomials Defined by Morgan-Voyce, Fibonacci Quart. 4 (1966), 73-81.

[25] M. N. S. Swamy, Further properties of Morgan-Voyce polynomials, Fibonacci Quart. 6 (1968), 167-175. MR0237470(38:5752)

Department of Mathematics and Statistics, University of North Carolina Charlotte, Charlotte, North Carolina 28223

E-mail address: ghetyei@uncc.edu 